\title{
Global prevalence of myasthenia gravis and the effectiveness of common drugs in its treatment: a systematic review and meta-analysis
}

\author{
Nader Salari', Behnaz Fatahi², Yalda Bartina ${ }^{3}$, Mohsen Kazeminia², Reza Fatahian ${ }^{4}$, Payam Mohammadi ${ }^{5}$,
} Shamarina Shohaimi ${ }^{6}$ and Masoud Mohammadi ${ }^{*}$ (1)

\begin{abstract}
Background: Myasthenia gravis is a neuromuscular autoimmune disorder characterized by weakness and disability in the voluntary muscles. There have been several preliminary studies on the epidemiology of myasthenia gravis in different parts of the world and the effectiveness of common drugs in its treatment, but there has been no comprehensive study of the efficacy of common drugs in the treatment of myasthenia gravis. Therefore, this study aimed to determine the epidemiology of myasthenia gravis globally and the effectiveness of common drugs in its treatment using systematic review and meta-analysis.
\end{abstract}

Methods: Research studies were extracted from IranDoc, MagIran, IranMedex, SID, ScienceDirect, Web of Sciences (WoS), ProQuest, Medline (PubMed), Scopus and Google Scholar based on Cochran's seven-step guidelines using existing keywords extracted in MeSH browser. The $\mathrm{I}^{2}$ test was used to calculate the heterogeneity of studies, and Begg and Mazumdar rank correlation tests were used to assess publication bias. Data were analyzed using Comprehensive Meta-Analysis software (Version 2).

Results: In the search for descriptive studies based on the research question, 7374 articles were found. After deleting articles unrelated to the research question, finally, 63 articles with a sample size of 1,206,961,907 people were included in the meta-analysis. The prevalence of MG worldwide was estimated to be 12.4 people $(95 \% \mathrm{Cl} 10.6-14.5)$ per 100,000 population. For analytical studies on the effectiveness of common myasthenia gravis drugs, 4672 articles were found initially, and after removing articles unrelated to the research question, finally, 20 articles with a sample size of 643 people in the drug group and 619 people in the placebo group were included in the study. As a result of the combination of studies, the difference between the mean QMGS score index after taking Mycophenolate and Immunoglobulin or plasma exchange drugs in the group of patients showed a significant decrease of $1.4 \pm 0.77$ and $0.62 \pm 0.28$, respectively $(P<0.01)$.

Conclusion: The results of systematic review of drug evaluation in patients with myasthenia gravis showed that Mycophenolate and Immunoglobulin or plasma exchange drugs have positive effects in the treatment of MG. It also represents the positive effect of immunoglobulin or plasma exchange on reducing SFEMG index and QMGS index

\footnotetext{
*Correspondence: Masoud.mohammadi1989@yahoo.com

${ }^{7}$ Cellular and Molecular Research Center, Gerash University of Medical Sciences, Gerash, Iran
}

Full list of author information is available at the end of the article permits use, sharing, adaptation, distribution and reproduction in any medium or format, as long as you give appropriate credit to the original author(s) and the source, provide a link to the Creative Commons licence, and indicate if changes were made. The images or other third party material in this article are included in the article's Creative Commons licence, unless indicated otherwise in a credit line to the material. If material is not included in the article's Creative Commons licence and your intended use is not permitted by statutory regulation or exceeds the permitted use, you will need to obtain permission directly from the copyright holder. To view a copy of this licence, visit http://creativecommons.org/licenses/by/4.0/. The Creative Commons Public Domain Dedication waiver (http://creativeco mmons.org/publicdomain/zero/1.0/) applies to the data made available in this article, unless otherwise stated in a credit line to the data. 
and the positive effect of Mycophenolate in reducing MG-ADL index, SFEMG and Anti-AChR antibodies index. In addition, based on a meta-analysis of the random-effect model, the overall prevalence of MG in the world is 12.4 people per 100,000 population, which indicates the urgent need for attention to this disease for prevention and treatment.

Keywords: Myasthenia gravis, MS, Prevalence, Drug, Systematic review, Meta-analysis

\section{Background}

Myasthenia gravis (MG) is a neuromuscular disease characterized by weakness in the voluntary muscles $[1,2]$. This disease has different symptoms that vary in different patients depending on the degree of involvement of the striated muscles. The most common type of symptom in patients with myasthenia gravis is ocular symptoms, which appear as ptosis and diplopia. These symptoms usually occur at the end of the day and follow activities such as watching TV or driving is more common, and excessive fatigue has been reported due to frequent activity in patients with this disease [3].

Myasthenia gravis is an autoimmune disease that connects the nerve to the muscle (NMJ) [4], which is produced by different antibodies against synaptic membrane proteins [5]. This is usually more than $85 \%$ of cases and is caused by a type of antibody against the skeletal muscle acetylcholine receptor (AChR-Ab) [6, 7]. However, components other than $\mathrm{AChR}$, such as muscle-specific tyrosine kinase receptor (MuSK) or lipoprotein-associated protein 4 (LRP4), may also be targeted for the autoimmune attack $[6,8,9]$.

Based on the mechanism of autoimmune disease and antibodies, invasive skeletal muscle molecules, thymus status, genetic characteristics, disease phenotype and response to treatment, myasthenia gravis is divided into early and late ocular subtypes (OMG), seronegative, thymoma, LRP4, MuSk. Diagnosis of MG subtype influences treatment decisions and disease prognosis $[10,11]$. Approximately $50 \%$ of patients with OMG develop general myasthenia gravis (GMG) over a 2-year period, which affects other muscles and manifests as weakness and ocular symptoms [12].

According to a systematic population-based study, CAR et al. [13] estimated the incidence and prevalence of MG at 54 per million and 77.7 per million, respectively. However, significant changes have been reported in various studies. The incidence of this disease has shown a range between 1.77 and 21.3 per million people and the prevalence of 15 to 179 million people [13]. A large number of epidemiological studies, mainly in Western Europe and Asia, reported significant differences in the incidence and prevalence of MG. The incidence of myasthenia gravis ranged from 1.7 to 30 per million per year [14-17].
The disease has two age peaks: age $40-40$ years, which mainly affects women, and another $80-60$ years, which occurs equally in men and women [4].

Current treatment options mainly include acetylcholinesterase inhibitors, glucocorticoids (GC), intravenous immunoglobulin (IVIg), plasma replacement (PLEX), thymectomy, and immunosuppressive agents including azathioprine prednisone, cyclosporine, cyclosporine, and cyclosporine [18-24]. However, the use of corticosteroids and immunosuppressants such as azathioprine manages MG. However, many patients do not tolerate or respond adequately to these drugs, and long-term treatment with $\mathrm{GC}$ is associated with a significant risk of side effects such as diabetes, obesity, and high blood pressure. This has led to the introduction of newer immunosuppressants such as mycophenolate mofetil (MMF) [12, 25-27].

About 15\% of patients with general myasthenia gravis do not respond to immunosuppressants and require intravenous immunoglobulin (IVIg) or plasma replacement (PLEX) to improve their symptoms [28, 29]. Intensified cases and myasthenia gravis crisis also require immediate treatment due to poor swallowing or respiratory failure that threatens the lives of these patients and muscle defects that may be a major disability for their daily activities [30].

Therefore, additional immunosuppression is often treated with Plasma Freesia (PLEX) and intravenous immunoglobulin (IVIg) to relieve symptoms. Plasma freeze uses filtration to kill pathological antibodies used in patients with myasthenia gravis and severe MG [31-33].

Myasthenia gravis has high direct health care costs (including long-term treatment and periodic hospitalization costs) and indirect costs such as loss of income and reduced care productivity [34]. Therefore, accurate identification of patients with MG is vital for organizing health care services and implementing preventive health measures. Many early articles have been done on the prevalence of myasthenia gravis and the effect of different drugs on the treatment process, but appropriate policy to control, diagnose and treat this disease requires coherent, accurate and uniform information. Therefore, the present study was performed to estimate the prevalence of myasthenia gravis globally and determine the effectiveness of the most common drugs in the treatment of patients by systematic review and meta-analysis. 


\section{Methods}

The present systematic review and meta-analysis were conducted according to the Cochrane seven-step approach, including selecting research questions, determining inclusion and exclusion criteria, identifying descriptive articles, selecting studies, qualitative evaluation of studies, data extraction, analysis and interpretation of findings [35].

\section{Research question and determining the keywords of the descriptive section}

According to the research question in the descriptive section, "How has the prevalence of MG in the world changed?" The population included: MG patients, Outcome included MG prevalence, Time or duration Included: Date of publication of the first related article until 15 November 2020 and type of study (study design) Included: cross-sectional (descriptive) studies. Keywords were extracted from the MeSH browser. Keywords related to the studied population (P): Myasthenia Gravis, MG and outcome keywords (O); Prevalence and Epidemiology.

\section{Research question and determining the keywords of the analytical section}

According to the research question of the analytical section, "What is the effectiveness of Corticosteroids, Mycophenolate and Immunoglobulin or plasma exchange drugs in the treatment of MG?" According to the PICO guidelines, the study population (Population) includes patients with $\mathrm{MG}$, intervention (intervention) including Corticosteroids, Mycophenolate and Immunoglobulin or plasma exchange, analogous (Comparison) including QMGS index score, Anti-AChR antibodies, SFEMG And MG-ADL before and after the intervention, the outcome (Outcome) included: changes in QMGS, Anti-AChR antibodies, SFEMG and MG-ADL after the intervention. Keywords were extracted from the MeSH browser according to PICO instructions. Keywords related to the study population (P): Myasthenia Gravis, MG Keywords related to the intervention (I); Corticosteroids, Corticotropin, Alternate-day prednisone, Methylprednisone, Prednisolone, Mycophenolate, Immunoglobulin or plasma exchange, Intravenous immunoglobulin, IVIG and keywords related to analogy $(\mathrm{C})$ and outcome $(\mathrm{O})$; QMGS, QMG, Anti-AChRantibodies, Anti-AChR ab, SFEMG and MG-ADL.

\section{Criteria for inclusion and exclusion according to the descriptive research question}

Cross-sectional (descriptive) studies in chronic patients reporting the prevalence of MG in different parts of the world published in English, and the full text was available. Observational studies, cohort, case-control, analytical and interventional studies, case reports, short reports, letters to the editor and studies unrelated to the research question were excluded from the study.

\section{Criteria for inclusion and exclusion according to the research question of the analytical section}

Clinical trial studies that reported the mean and standard deviation of the effect of Corticosteroids, Mycophenolate and Immunoglobulin or plasma exchange on at least one of the indicators of QMGS, Anti-AChR antibodies, SFEMG and MG-ADL in patients with MG, in Persian and were printed in English and their full text was available and included in the study. Descriptive studies, crosssectional studies, reviews, case reports, short reports, letters to the editor, and other studies unrelated to the research question were excluded from the study.

\section{Articles identification}

To find studies related to research questions, four Persian databases, including IranDoc, MagIran, IranMedex and SID and five international databases: ScienceDirect, Web of Science (WoS), ProQuest, Medline (PubMed), Scopus were searched. The Google Scholar scientific search engine was reviewed for final review. No time limit was set for the search to retrieve the relevant research, so all articles published by November 15, 2020, were reviewed. The search was limited to studies published in Persian and English. The search strategy in each database was determined using Advanced Search (Advanced Search) with the help of all possible keyword combinations with the help of (AND) and (OR) operators. For example, the search strategy in the PubMed database for the descriptive part of the research was determined as follows:

(((Prevalence [Title/Abstract]) OR (Epidemiology [Title/Abstract])) AND (Myasthenia Gravis [Title/ Abstract])) OR (chronic patients [Title/Abstract]) OR (MG [Title/Abstract]).

Also, the search strategy in the PubMed database for the analytical part of the research was determined as follows:

()(()(()(()(()(Corticosteroids[Title/Abstract]) $\quad$ OR (Corticotropin[Title/Abstract])) OR (Alternate-day prednisone[Title/Abstract])) OR (Methylprednisone [Title/ Abstract])) OR (chronic patients [Title/Abstract]) OR (Prednisolone[Title/Abstract])) OR (Mycophenolate[Title/ Abstract])) OR (Immunoglobulin[Title/Abstract] OR plasma exchange[Title/Abstract])) OR (Intravenous immunoglobulin[Title/Abstract])) OR (IVIG[Title/ Abstract])) AND (QMGS[Title/Abstract])) OR (QMG[Title/ Abstract])) OR (Anti-AChR antibodies[Title/Abstract])) OR (Anti-AChR ab[Title/Abstract])) OR (SFEMG[Title/ Abstract])) OR (MG-ADL[Title/Abstract])) AND (Myasthenia Gravis[Title/Abstract])) OR (MG[Title/Abstract]). 
In order to access the latest published studies, an alert was created on several databases, including PubMed and Scopus, to check if new articles were published during the study. Also, in order to access all related studies, the sources of articles that met the inclusion criteria were manually reviewed. To avoid errors, all steps of article search, study selection, qualitative evaluation and data extraction were performed independently by two researchers (M.K. and B.F.). If there was a difference of opinion between the researchers regarding the inclusion of the article in the study, in order to avoid the risk of bias for specific studies, first a final agreement was reached through discussion and in some cases with the participation and opinion of a third party (MM).

\section{Selection of studies based on entry and exit criteria}

Based on the 4-step PRISMA process, including article identification, screening, eligibility, and inclusion in the study, three researchers reviewed this process as follows, and studies were selected based on inclusion and exclusion criteria. All articles found in each database were transferred to EndNote X8 software. After completing the search in all the databases, the articles repeated in different databases were deleted. Then, in order to avoid the risk of bias in selecting studies, the names of the authors and the titles of the journals were removed, and a checklist was prepared based on the titles and abstracts of the studies. In the next step, two authors (M.K. and B.F.) independently examined the title and abstract of the studies and eliminated studies that were not related to the research based on the inclusion and exclusion criteria of the study and in case of discrepancy, it was examined by the third researcher (M.M). Studies whose full text was not found were also excluded from the systematic review and meta-analysis process. The full text of all remaining articles was then evaluated. Studies that did not meet the inclusion criteria based on the research question were excluded.

\section{Qualitative evaluation of descriptive studies}

Qualitative evaluation of studies was performed using the STROBE checklist, a suitable tool for the qualitative assessment of descriptive studies. This checklist has 22 general items, each of which has sub-items (32 subitems in total) and to evaluate different parts of a study, including title and abstract, study objectives, problem statement, study type, sampling method, study statistical population, the sample size is the definition of variables, tools for collecting study data, statistical analysis, findings and discussion. In order to rate the articles, if each article referred to the items considered in the checklist, it was given a score of 1 , and if it was not mentioned, a score of zero was given. The minimum and maximum scores in this checklist are 0 and 32, respectively. Articles with scores of 16 and above were considered high and medium quality studies and were included in the systematic review and meta-analysis process, and articles with scores below 16 were considered low-quality studies [36].

\section{Qualitative evaluation of analytical studies}

Qualitative evaluation of studies was performed using the CONSORT checklist, a suitable tool for the qualitative assessment of interventional studies. This checklist has 25 general items, each with minor items (a total of 37 minor items). Different sections include Title and Abstract, Introduction and Background, Methods, Participants, Interventions, Objectives, Consequences, Sample Size, Randomization, How to Assign Participants, Blind Allocation, Execution, Blindness of Study, Statistical Methods, Results, Flow Participants' presence, sampling method, initial data of the number of people analyzed, consequences and estimates, auxiliary analysis, adverse reactions, explanations, interpretation, generalizability and general evidence. In this study, all general checklist items were reviewed by two authors (M.K. and B.F.). In order to rate the articles, if each article referred to the items in the checklist, it was given a score of 1 , and if it was not mentioned, a score of zero was given. The minimum and maximum scores in this checklist are 0 and 37 , respectively. Studies with $75 \%$ or more of the maximum achievable score (score greater than or equal to 27) with "high quality", studies with a score between 75 and $50 \%$ (score 18-26) as "average quality" and studies with a score below $50 \%$ (less than or equal to 17 ) were considered "low quality" studies [37]. Based on this checklist, medium and high-quality articles were included in the study, and low-quality articles were excluded.

\section{Data extraction}

After selecting the studies to enter the systematic review and meta-analysis process, the data were extracted, and the studies were summarized. For this purpose, two electronic checklists (one for the descriptive section and one for the analytical section) were prepared. The various items in the descriptive checklist included: name of the first author, year of publication and year of the report, place of study, age, sample size and prevalence, and various items in the analytical checklist, including the name of the first author, year of publication, place of research, the sample size of the drug group and the placebo group was the type of drug, mean and standard deviation before and after the intervention. 


\section{Statistical analysis of the descriptive part}

To analyze and combine the results of different studies, in each study, the prevalence of MG was considered as the probability of binomial distribution and its variance was calculated through binomial distribution. Heterogeneity of studies was assessed using the $\mathrm{I}^{2}$ test, and the random-effects model was used in the case of the $\mathrm{I}^{2}$ index above $50 \%$. In this model, parametric changes between studies are also considered in the calculations, so it can be said that the results of this model in heterogeneous conditions can be more generalized than the model with a fixed effect. Publication bias assessment was performed using Funnel Plot and Begg and Mazumdar rank correlation test. Data were analyzed using Comprehensive Meta-Analysis (Version 2) software, and the significance level of the test was $\mathrm{P}<0.05$.

\section{Statistical analysis of the analytical section}

In this study, the standardized mean difference was calculated. The main outcome of this study was the mean score of the studied indicators before and after the intervention in patients with MG. As a result, the mean and standard deviation of the studied indices before and after the intervention were extracted. $\mathrm{I}^{2}$ index was used to evaluate heterogeneity. Funnel Plot and Begg and Mazumdar rank correlation tests were used to assess the publication bias. The significance level of the test was considered 0.1. Data were analyzed using Comprehensive Meta-Analysis software (Version 2).

\section{Results}

\section{Descriptive part of the study}

Summary of how articles enter meta-analysis: In the first stage, 7374 articles (7192 articles in international databases, 159 articles in Persian databases and 23 studies in reviewing article sources) were found, of which 5368 studies were repeated in different databases were removed. A total of 2006 studies were entered the in the screening stage and 1851 articles were deleted based on the inclusion and exclusion criteria by reviewing the title and abstract of the study. In the next stage (competency assessment), out of the remaining 155 studies from the screening stage, 92 articles were removed by reviewing the full text of the article because it was not relevant to the research. The quality evaluation of 63 articles included in this study was performed using the STROBE checklist, all of which were of medium and high quality according to the criteria of this tool. Thus, 63 articles related to the descriptive part of the study entered the process of systematic review and metaanalysis (Fig. 1).

\section{General characteristics of the studies}

The total sample size of the studies was $1,206,961,907$ people. The studies were published between 1969 and November 15, 2020. There were 8 studies in Asia, 42 in Europe, 7 in the United States, 5 in Africa, and 1 in Australia. Summary of study characteristics, including the name of the first author, year of publication and year of the report, place of study, mean age of patients, sample size and prevalence of MG, are reported in Table 1.

\section{Systematic meta-analysis and descriptive review}

The result of the $\mathrm{I}^{2}$ test for the prevalence of MG in the world indicates a significant heterogeneity between studies $\left(\mathrm{I}^{2}=99.9\right)$, so the data were analyzed by meta-analysis using a random-effects model. Due to the high heterogeneity of the studies, sensitivity analysis was performed, and each study's effect on the final result and the degree of heterogeneity was evaluated. None $(\mathrm{P}=0.103)$ (Fig. 2). As a result of the combination of studies, the overall estimate of the global prevalence of MG $1^{2}$. 4 people $(95 \%$ confidence interval: $10-14-5.5)$ per 100,000 population was based on a random-effects model. The black square is the prevalence and the length of the line segment on which the $95 \%$ confidence interval per It is a study, the rhombus symbol shows the worldwide prevalence for all studies (Fig. 3). The highest prevalence was reported in Salvado et al. [61]; 3463 per 100,000 population and the lowest prevalence Bettini et al. [58]; 0.006 people reported per 100,000 population.

According to different reports of MG prevalence in different parts of the world, subgroup analysis by different continents (Asia, Europe, Africa and America) is reported in Table 2, which has the highest prevalence in the Americas with 19 people (95\% CI 15-23.8) (Table 2).

\section{The analytical part of the study}

Summary of how to enter articles: In the first stage, 4672 articles (4596 articles in international databases, 45 articles in Persian databases and 31 studies in reviewing the sources of articles) were found, and 3126 studies that were repeated in different databases were deleted. 1546 studies were entered in the screening stage, and based on the inclusion and exclusion criteria, the article was removed by reviewing the title and abstract of the 1992 studies. In the next stage (competency assessment), out of the remaining 175 studies from the screening stage, 183 articles were removed by reviewing the full text of the article because it was not relevant to the research. The remaining 22 articles were evaluated qualitatively by the CONSORT checklist, of which 2 studies were of low quality according to the criteria of this tool and were 


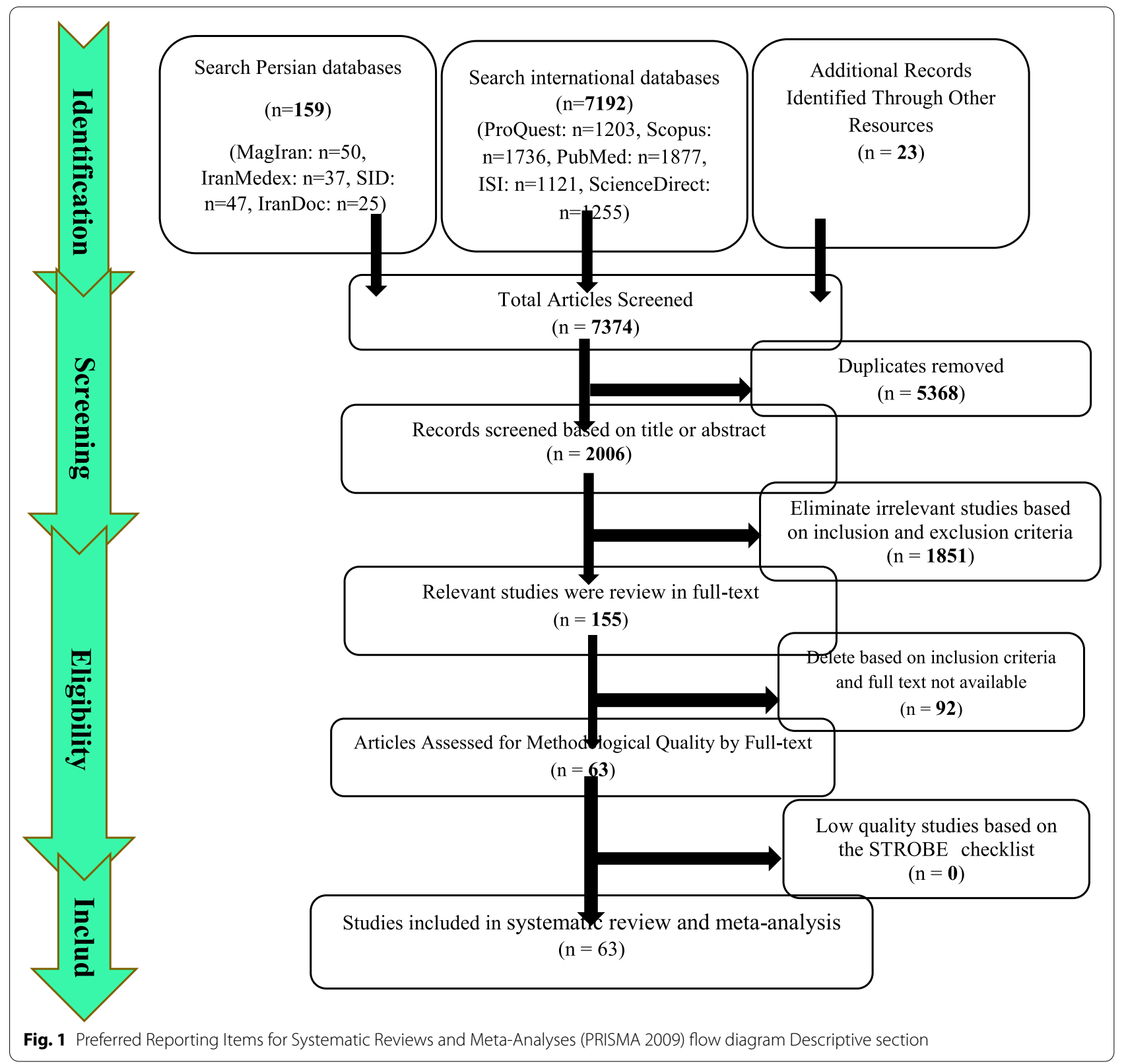

excluded from the study. Therefore, 20 articles related to the analytical part of the study were included in the systematic review and meta-analysis process (Fig. 4).

\section{General characteristics of analytical studies}

The total sample size was 643 in the drug group and 619 in the placebo group. The studies were published between 1976 and November 15, 2020. The smallest sample size was related to the study of Benatar et al. [109] with 6 patients in the drug group and 5 patients in the placebo group, and the largest sample size was related to the study of Sanders et al. [112] with 88 patients in the drug group and 88 patients in the placebo group. Summary of study characteristics including the name of the first author, year of publication, place of study, sample size, type of drug, and mean and standard deviation before and after the intervention of QMGS, Anti-AchR antibodies, SFEMG and MG-ADL indices are reported in Table 3.

\section{Immunoglobulin or plasma exchange drugs}

A total of 13 studies examined the effect of immunoglobulin or plasma exchange drugs on MG patients. Studies were reported from 1997 to 2020. 11 studies 
Table 1 General characteristics of descriptive studies

\begin{tabular}{|c|c|c|c|c|c|}
\hline First Author, year, Reference & Report year & Country & Age (years) & Sample size & $\begin{array}{l}\text { Prevalence per } \\
\text { hundred thousand } \\
\text { people }\end{array}$ \\
\hline Murai-1, 2011, [38] & 1987 & Japan & - & $117,647,059$ & 5.09 \\
\hline Murai-2, 2011, [38] & 2006 & Japan & - & $127,966,102$ & 11.79 \\
\hline Nemet-1, 2014, [39] & 2014 & Israel & - & 475,097 & 46.93 \\
\hline Nemet-2, 2014, [39] & 2014 & Israel & - & 52,160 & 47.92 \\
\hline Park-1, 2016, [40] & 2010 & Korea & - & $50,744,570$ & 9.67 \\
\hline Park-2, 2016, [40] & 2011 & Korea & - & $50,750,469$ & 10.66 \\
\hline Lee-1, 2016, [41] & 2010 & Korea & - & $47,990,761$ & 10.42 \\
\hline Lee-2, 2016, [41] & 2011 & Korea & - & $49,779,440$ & 11.03 \\
\hline Lee-3, 2016, [41] & 2012 & Korea & - & $50,004,441$ & 12.4 \\
\hline Lee-4, 2016, [41] & 2013 & Korea & - & $50,219,669$ & 12.96 \\
\hline Lee-5, 2016, [41] & 2014 & Korea & - & $50,423,955$ & 12.99 \\
\hline Okinaka, 1969, [42] & 1966 & Japan & - & 838,000 & 1.78 \\
\hline Araki, 1983, [43] & 1982 & Japan & - & 537,313 & 6.7 \\
\hline Kondo, 1988, [44] & 1985 & Pakistan & - & $1,038,462$ & 2.59 \\
\hline Yu, 1992, [45] & 1987 & China & - & $4,860,000$ & 5.34 \\
\hline Zieda, 2018, [46] & 2018 & UK & 46 & 1986096 & 11.37 \\
\hline Lavrnic-1, 2013, [47] & 2013 & Serbia & $47.9 \pm 19.8$ & $1,338,161$ & 31.76 \\
\hline Tola, 1989, [48] & 1989 & Italy & $47.8 \pm 18.1$ & 370,374 & 10.52 \\
\hline Montomoli, 2012, [49] & 2012 & Italy & $58.3 \pm 17.0$ & 495,833 & 24 \\
\hline Cetin, 2012, [50] & 2012 & Austria & - & $8,363,040$ & 7.99 \\
\hline Storm-1, 1984, [51] & 1951 & Norway & - & $3,100,000$ & 2 \\
\hline Storm-2, 1984, [51] & 1961 & Norway & - & $3,463,415$ & 4.09 \\
\hline Storm-3, 1984, [51] & 1971 & Norway & - & $3,794,872$ & 9.98 \\
\hline Storm-4, 1984, [51] & 1981 & Norway & - & $4,107,063$ & 8.98 \\
\hline Westerberg, 2020, [52] & 2020 & Sweden & 60 & $13,119,113$ & 36.1 \\
\hline Kalb, 2002, [53] & 2002 & Sweden & & $1,783,428$ & 14.07 \\
\hline Aiello, 1997, [54] & 1997 & Italy & $44 \pm 16.6$ & 268,926 & 11.15 \\
\hline Guidetti, 1998, [55] & 1998 & Italy & $50.5 \pm 19.8$ & 427,493 & 10.29 \\
\hline Foldvari, 2015, [56] & 2015 & Hungary & 60 & $8,259,048$ & 2.76 \\
\hline Zivadinov, 1998, [57] & 1998 & Italy & $67.7-139.6$ & 323,232 & 9.9 \\
\hline Bettini, 2017, [58] & 2017 & Argentina & $63.3 \pm 20$ & $978,313.561$ & 0.006 \\
\hline Andersen, 2014, [59] & 2014 & Norway & - & $4,725,190$ & 13.1 \\
\hline Aragonès, 2017, [60] & 2017 & Spain & - & 155,062 & 32.89 \\
\hline Salvado, 2016, [61] & 2016 & Spain & $59.44 \pm 29.35$ & 462 & 3463 \\
\hline Christensen-1, 1993, [62] & 1993 & Denmark & - & $280,000,000$ & 7.8 \\
\hline Robertson, 1998, [63] & 1998 & England & - & 684,000 & 14.6 \\
\hline Garland, 1956, [64] & 1955 & UK & - & 500,000 & 3.6 \\
\hline Pennington, 1961, [65] & 1958 & UK & - & $1,500,000$ & 2.13 \\
\hline Gudmundsson, 1968, [66] & 1963 & Iceland & - & 187,000 & 6.41 \\
\hline Oosterhuis, 1977, [67] & 1965 & Holland & - & 860,000 & 5.58 \\
\hline Hokkanen, 1969, [68] & 1968 & Finland & - & $4,493,392$ & 2.55 \\
\hline Giagheddu, 1989, [69] & 1986 & Italy & - & $2,444,444$ & 4.5 \\
\hline D'Alessamdro-1, 1991, [70] & 1987 & Italy & - & 914,463 & 7.21 \\
\hline D'Alessamdro-2, 1991, [70] & 1988 & Italy & - & 370,374 & 10.52 \\
\hline Sorensen, 1989, [71] & 1987 & Denmark & - & 230,760 & 12.56 \\
\hline Somnier-1, 1991, [72] & 1988 & Eastern Denmark & - & $2,298,701$ & 17.66 \\
\hline Christensen-2, 1998, [73] & 1990 & Western Denmark & - & $2,800,000$ & 7.85 \\
\hline Ferrari, 1992, [74] & 1990 & Italy & - & 446,914 & 8.27 \\
\hline
\end{tabular}


Table 1 (continued)

\begin{tabular}{|c|c|c|c|c|c|}
\hline First Author, year, Reference & Report year & Country & Age (years) & Sample size & $\begin{array}{l}\text { Prevalence per } \\
\text { hundred thousand } \\
\text { people }\end{array}$ \\
\hline Krivopusk, 1991, [75] & 1991 & Russia & - & 655,738 & 3.5 \\
\hline Lavrnic-2, 1999, [76] & 1992 & Serbia & - & $1,530,864$ & 7.64 \\
\hline Kyriallis, 1995, [77] & 1994 & Cyprus & - & 600,000 & 17.5 \\
\hline Holtsema, 2000, [78] & 1995 & Netherlands Antilles & - & 229,800 & 6.52 \\
\hline Villagr, 1997, [79] & 1996 & Spain & - & 81,507 & 8.58 \\
\hline Oopik, 2003, [80] & 1997 & Estonia & - & $1,462,130$ & 14.22 \\
\hline Wirtz, 2003, [81] & 2000 & Southern Holland & - & $1,725,317$ & 10.95 \\
\hline Kotov-1, 2006, [82] & 2001 & Southern Holland & - & $12,000,000$ & 8.96 \\
\hline Kotov-2, 2006, [82] & 2004 & Southern Holland & - & $1,778,564$ & 14.22 \\
\hline Eaton, 2007, [17] & 2001 & Denmark & - & $5,472,032$ & 17.85 \\
\hline Somnier-2, 2005, [83] & 1999 & Eastern Denmark & - & $2,298,701$ & 16.35 \\
\hline Poulas, 2001, [84] & 1997 & Greece & - & $10,475,873$ & 7.06 \\
\hline Niks-1, 2007, [85] & 2004 & Netherlands & - & $1,778,564$ & 8.99 \\
\hline Niks-2, 2007, [85] & 2004 & Netherlands & - & $1,778,564$ & 0.28 \\
\hline Tsiamalos, 2009, [86] & 2006 & Greece & - & $11,293,282$ & 0.29 \\
\hline Breiner-1, 2016, [15] & 1996 & Canada & - & $8,586,605$ & 16.56 \\
\hline Breiner-2, 2016, [15] & 1997 & Canada & - & $8,734,231$ & 17.79 \\
\hline Breiner-3, 2016, [15] & 1998 & Canada & - & $8,884,264$ & 18.79 \\
\hline Breiner-4, 2016, [15] & 1999 & Canada & - & $9,055,208$ & 19.86 \\
\hline Breiner-5, 2016, [15] & 2000 & Canada & - & $9,247,809$ & 20.79 \\
\hline Breiner-6, 2016, [15] & 2001 & Canada & - & $9,453,075$ & 21.59 \\
\hline Breiner-7, 2016, [15] & 2002 & Canada & - & $9,644,864$ & 22.19 \\
\hline Breiner-8, 2016, [15] & 2003 & Canada & - & $9,825,322$ & 22.88 \\
\hline Breiner-9, 2016, [15] & 2004 & Canada & - & $10,004,779$ & 23.79 \\
\hline Breiner-10, 2016, [15] & 2005 & Canada & - & $10,173,985$ & 24.69 \\
\hline Breiner-11, 2016, [15] & 2006 & Canada & - & $10,189,022$ & 26.09 \\
\hline Breiner-12, 2016, [15] & 2007 & Canada & - & $10,173,121$ & 26.79 \\
\hline Breiner-13, 2016, [15] & 2008 & Canada & - & $10,346,890$ & 28.09 \\
\hline Breiner-14, 2016, [15] & 2009 & Canada & - & $10,535,185$ & 29.19 \\
\hline Breiner-15, 2016, [15] & 2010 & Canada & - & $10,723,870$ & 25.49 \\
\hline Breiner-16, 2016, [15] & 2011 & Canada & - & $10,923,120$ & 31.19 \\
\hline Breiner-17, 2016, [15] & 2012 & Canada & - & $11,114,448$ & 31.89 \\
\hline Breiner-18, 2016, [15] & 2013 & Canada & - & $11,274,236$ & 32.02 \\
\hline Maharaj, 2013, [87] & 2013 & USA & - & 412,810 & 8.72 \\
\hline Gordon, 2015, [88] & 2015 & USA & - & 265,844 & 77 \\
\hline Phillips-1, 1992, [89] & 1980 & USA & - & 537,313 & 13.4 \\
\hline Phillips-2, 1992, [89] & 1984 & USA & - & 556,338 & 14.2 \\
\hline Kurland, 1958, [90] & 1954 & USA & - & 30,000 & 3.33 \\
\hline Alter, 1960, [91] & 1956 & USA & - & 188,000 & 3.19 \\
\hline Kvirkveliia, 1986, [92] & 1984 & USA & - & 555,851 & 14.2 \\
\hline Cisernos, 1996, [93] & 1991 & Cuba & - & $5,782,309$ & 2.92 \\
\hline Sanchez, 2002, [94] & 2000 & Colombia & - & $5,300,000$ & 2.77 \\
\hline Deffeminis, 1975, [95] & 1975 & Uruguay & - & $2,700,000$ & 6.29 \\
\hline Khedr, 2016, [96] & 2016 & Qena & - & 9303 & 21.49 \\
\hline El-Tallawy, 2005, [97] & 1997 & Egypt & - & 50,000 & 10 \\
\hline Gattellari, 2012, [98] & 2012 & Australia & - & 21874920 & 2.49 \\
\hline
\end{tabular}




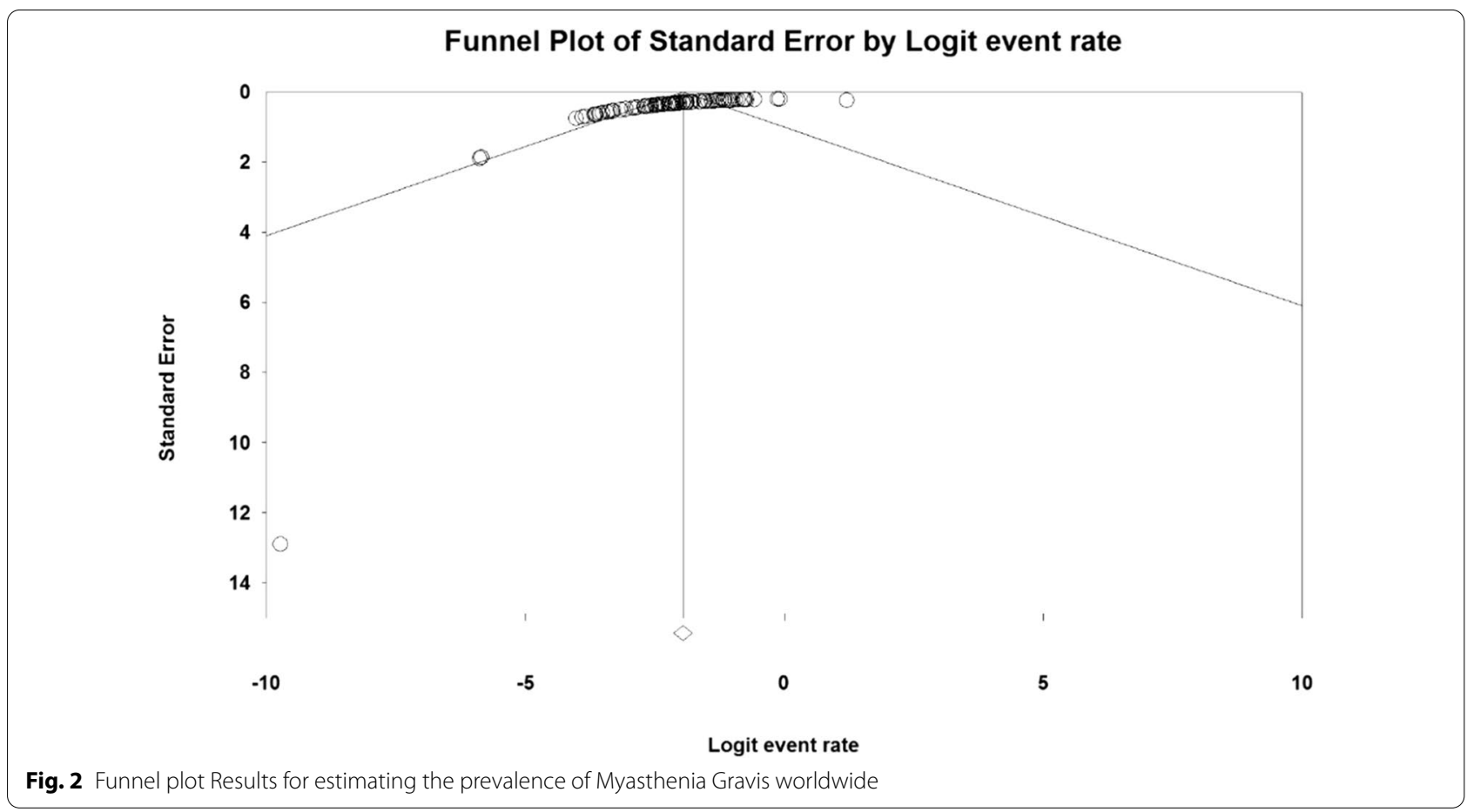

examined the QMGS index, 4 studies the Anti-AChR antibodies, 4 studies the SFEMG index, and 3 the MGADL index.

\section{MG-ADL index}

The Daily Living Activity Scale (MG-ADL) is an 8-item scale to assess secondary ocular disability (two items), bulbar (three items), respiratory (one item), limb (two items) related to myasthenia gravis effects. This scale has a linear rating from zero to 3 , and its overall scoring range is from zero to 24 [115]. The patient's MG-ADL questionnaire is completely reported without training and specialized equipment and usually lasts less than five minutes [116].

Based on the present meta-analysis results between studies, there is a lot of heterogeneity $\left(\mathrm{I}^{2}=78.5\right)$, so the stochastic effects model was used to combine the studies and the final result. Begg and Mazumdar rank correlation to test the publication bias in the studies for the MGADL index $(\mathrm{P}=1.000)$ (Table 4).

As a result of the combination of studies, the mean score of MG-ADL indices before the intervention in the drug group was $8.2 \pm 1.3$. After the intervention was $4.0 \pm 4.84$ (Table 4), as well as the difference between the mean of the MG-ADL index before and after the intervention, $1.3 \pm 0.63$ was obtained $(\mathrm{P}<0.01)$ (Fig. 5), which indicates the positive effect of Immunoglobulin or plasma exchange on the reduction of MG-ADL index.

\section{SFEMG index}

Single-fiber electromyography (SFEMG) is an efficient tool to investigate neurotransmitter disorders. In this method, with the help of bipolar needle electrodes, the action potential of two adjacent muscle fibres belonging to a motor unit that have been activated voluntarily or electrically stimulated can be recorded [117]. This technique is more time consuming than conventional EMG, and patient cooperation in this method is effective because even small movements by the patient can lead to loss or change of action potential [118].

Based on the present meta-analysis results between studies, there is a lot of heterogeneity $\left(\mathrm{I}^{2}=43.5\right)$, so the stochastic effects model was used to combine the studies and the final result. Begg and Mazumdar rank correlation tests were not available in studies for the SFEMG index $(\mathrm{P}=1.000)$ (Table 4). As a result of the combination of studies, the mean score of SFEMG indices before the intervention in the drug group was $82.2 \pm 1.43$, and after the intervention was $54 / 5 \pm 27 / 5$ (Table 4), also, the difference between the mean of SFEMG index before and after the intervention was $1.5 \pm 0.73(\mathrm{P}<0.01)$ (Fig. 6), which indicates the positive effect of immunoglobulin or plasma exchange on the reduction of SFEMG index.

\section{Anti-AChR antibodies index}

Weakness and fatigue in myasthenia gravis are caused by a decrease in acetylcholine receptors due to an 


\section{Meta Analysis}

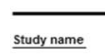

Statistics for each study

Event rate and $95 \% \mathrm{Cl}$

$\begin{gathered}\text { Event } \\ \text { rate }\end{gathered}$
limit

$\begin{array}{llllll}\text { Murati-1 } & 0.051 & 0.022 & 0.116 & 6.430 . & 0.000 \\ \text { Muraia-2 } & 0.118 & 0.068 & 0.197 & 6.490- & 0.000 \\ \text { Nemet-1 } & 0.469 & 0.374 & 0.567 & 0.614 . & 0.539\end{array}$

$\begin{array}{lllllll}\text { Nemet-1 } & 0.469 & 0.374 & 0.567 & 0.614- & 0.539 \\ \text { Nemet-2 } & 0.479 & 0.383 & 0.577 & 0.416- & 0.577\end{array}$

$\begin{array}{llllll}\text { Park-1 } & 0.479 & 0.383 & 0.577 & 0.416- & 0.677 \\ \text { Park-2 } & 0.097 & 0.052 & 0.172 & 6.604 . & 0.000 \\ & 0.107 & 0.059 & 0.184 & 6.561 . & 0.000\end{array}$

$\begin{array}{llllll}\text { Lee-1 } & 0.104 & 0.058 & 0.181 & 6.573 . & 0.0000 \\ \text { Lee. } & 0.110 & 0.062 & 0.000 & 0.542 & 0.000\end{array}$

$\begin{array}{llllll}\text { Lee-2 } & 0.110 & 0.062 & 0.188 & 6.540 . & 0.000 \\ \text { Lee-3 } & 0.124 & 0.072 & 0.204 & 6.444 . & 0.000 \\ \text { Lee-4 } & 0.130 & 0.077 & 0.211 & 6.397 . & 0.000\end{array}$

$\begin{array}{llllll}\text { Lee-4 } & 0.130 & 0.077 & 0.211 & 6.397- & 0.000 \\ \text { Lee-5 } & 0.130 & 0.077 & 0.211 & 6.394- & 0.000 \\ \text { Oknaka } & 0.018 & 0.004 & 0.074 & 5303- & 0\end{array}$

$\begin{array}{llllll}\text { Oknaka } & 0.018 & 0.004 & 0.074 & 5.303- & 0.000 \\ \text { Araki } & 0.067 & 0.032 & 0.136 & 6.585- & 0.000 \\ \text { Kondo } & 0.026 & 0.008 & 0.084 & 5.761 & 0.000\end{array}$

$\begin{array}{llllll}\text { Kondo } & 0.026 & 0.008 & 0.084 & 5.761 . & 0.000 \\ \text { YuYL } & 0.053 & 0.023 & 0.119 & 6.464 . & 0.000 \\ \text { Zieda } & 0.114 & 0.065 & 0.192 & 6519 . & 0.000\end{array}$

$\begin{array}{llllll}\text { Zleda } & 0.114 & 0.065 & 0.192 & 6.519- & 0.000 \\ \text { Lavrnic } & 0.318 & 0.234 & 0.415 & 3.561- & 0.000 \\ \text { Tola } & 0.105 & 0.058 & 0.182 & 6.568- & 0.000\end{array}$

$\begin{array}{llllll} & 0.105 & 0.058 & 0.182 & 6.562- & 0.000 \\ \text { Muontomali } & 0.240 & 0.166 & 0.333 & 4.923 . & 0.000 \\ \text { Cetin } & 0.080 & 0.040 & 0.152 & 6.626- & 0.000 \\ & 0.020 & 0.005 & 0.070 & 5.42 & 0.000\end{array}$

$\begin{array}{llllll}\text { Storm-1 } & 0.020 & 0.005 & 0.076 & 5.449 . & 0.000 \\ \text { Storm-2 } & 0.041 & 0.016 & 0.103 & 6.248- & 0.000 \\ \text { Strom-3 } & 0.100 & 0.055 & 0.170 & 0.92 .2 & 0.000\end{array}$

\begin{tabular}{llllll} 
Storm-3 & 0.100 & 0.055 & 0.176 & $6.592-$ & 0.000 \\
\hline Stom-4 & 0.090 & 0.047 & 0.04 & 0.22. & 0.000
\end{tabular}

$\begin{array}{llllll}\text { Storm-4 } & 0.090 & 0.047 & 0.164 & 6.622 . & 0.000 \\ \text { Westerberg } & 0.361 & 0.273 & 0.459 & 2.743 . & 0.006 \\ & 0.112 & 0.005 & 0.19 & 0.52 & 0.000\end{array}$

\begin{tabular}{llllll} 
Kalb & 0.141 & 0.085 & 0.223 & 6.292. & 0.0000 \\
Aiello & 0.112 & 0.063 & 0.190 & 6.533. & 0.000 \\
\hline & 0.103 & 0.005 & 0.029 & 5035 & 0.000
\end{tabular}

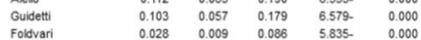

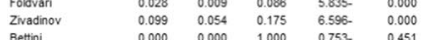

\begin{tabular}{llllll}
\hline Andersen & 0.131 & 0.078 & 0.212 & 6.384 & 0.051 \\
\hline & 0.000 & 0.000
\end{tabular}

$\begin{array}{llllll}\text { Aragonens } & 0.329 & 0.244 & 0.427 & 3.351 . & 0.001 \\ \text { Christensen } & 0.078 & 0.039 & 0.149 & 6.623 . & 0.000\end{array}$

$\begin{array}{llllll}\text { Christensen } & 0.078 & 0.039 & 0.149 & 6.623- & 0.000 \\ \text { Robertson } & 0.146 & 0.089 & 0.229 & 6.237 . & 0.000\end{array}$

$\begin{array}{llllll}\text { Garland } & 0.036 & 0.013 & 0.097 & 6.237 . & 0.000 \\ & & 0.000\end{array}$

$\begin{array}{llllll}\text { Pennington } & 0.021 & 0.006 & 0.078 & 5.526- & 0.000 \\ \text { Gudmundsson } & 0.064 & 0.030 & 0.132 & 6567 . & 0.000\end{array}$

\begin{tabular}{llllll} 
Oostertuis & 0.056 & 0.025 & 0.122 & $6.4933-$ & 0.0000 \\
\hline & 0.004 & 0.007 & 0.10 & 635 & 0.000
\end{tabular}

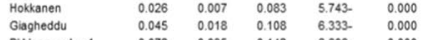

$\begin{array}{lllllll}0.072 & 0.035 & 0.142 & 6.608 & 0.000\end{array}$

$\begin{array}{llllll}\text { DAlessamdro-2 } & 0.105 & 0.058 & 0.182 & 6.568 \text { - } & 0.000 \\ \text { Sorensen } & 0.126 & 0.074 & 0.206 & 6.431 . & 0.000\end{array}$

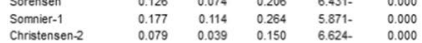

$\begin{array}{llllll}\text { Christensen-2 } & 0.079 & 0.039 & 0.150 & 6.624- & 0.000 \\ \text { Ferrari } & 0.083 & 0.042 & 0.155 & 6.627 . & 0.000\end{array}$

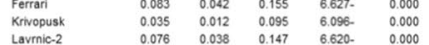

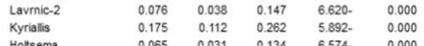

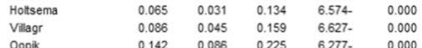

$\begin{array}{llllll} & 0.142 & 0.086 & 0.225 & 6.277 . & 0.000 \\ \text { Woptz } & 0.110 & 0.062 & 0.187 & 6.545 . & 0.000 \\ \text { Kottov-1 } & 0.090 & 0.047 & 0.164 & 6.622 . & 0.000\end{array}$

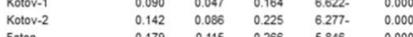

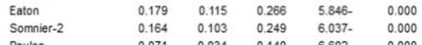

Poulas
Nilks-1

Niks-2

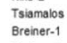

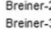

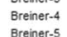

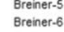

Breiner-7
Breiner-8

Brenene-9
Brener-10

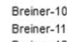

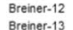

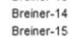

Brener-16

Brenerer-17
Brener-18

$\begin{array}{lllll}0.320 & 0.235 & 0.416 & 3.537- & 0.000 \\ 0.236 & 0.418 & 3.512 . & 0.000 \\ 0.877 & 0.046 & 0.181 & 0.255 & 0.000\end{array}$

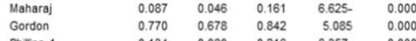

$\begin{array}{lllllll}\text { Phillps-1 } & 0.134 & 0.080 & 0.216 & 6.0357 . & 0.0000 \\ \text { Phllps-2 } & 0.142 & 0.095 & 0.225 & 5279 & 0.000\end{array}$

\begin{tabular}{llllll} 
Phillps-2 & 0.142 & 0.026 & 0.225 & $6.279-$ & 0.000 \\
Kunland & 0.033 & 0.011 & 0.093 & 6.043. & 0.000 \\
\hline & 0.142 & 0.006 & 0.25 & 6279 & 0.000
\end{tabular}

Kvirkveliia
Cisernos
Sornotis

Sanchez

Deffeminis
Khedr

$\begin{array}{lllll}0.142 & 0.086 & 0.225 & 5.997 . & 0.000 \\ 0.279 . & 0.000\end{array}$

$\begin{array}{lllll}0.029 & 0.009 & 0.0258 & 6.279- & 0.000 \\ 0.099 . & 0.000 & 0.000\end{array}$

$\begin{array}{lllll}0.100 & 0.055 & 0.176 & 6.592 . & 0.000 \\ 0.025 & 0.007 & 0.082 & 5.715- & 0.000\end{array}$

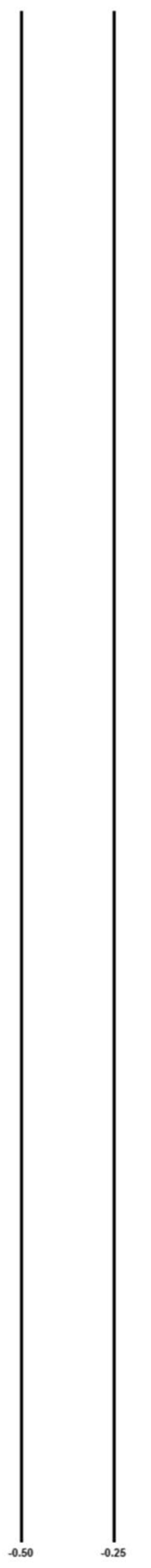

-

主

$=$

$=$

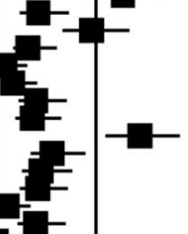

$+$

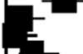

$=$
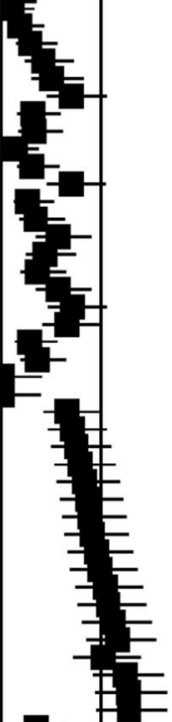

난

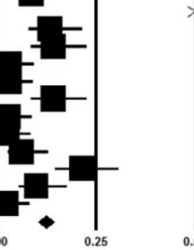

Favours 8

\section{Meta Analysis}

Fig. 3 Estimation of the prevalence of Myasthenia Gravis in the world based on a random-effects model 
Table 2 Prevalence of Myasthenia gravis by different continents

\begin{tabular}{llllll}
\hline Continents & Number of articles & Sample size & $\mathbf{I}^{\mathbf{2}}$ & $\begin{array}{l}\text { Begg and mazumdar rank } \\
\text { correlation test }\end{array}$ & Prevalence per 100,000 \\
\hline Asia & 15 & $603,327,498$ & 91.8 & 0.101 & $10.9(95 \% \mathrm{Cl} 6.4-17.9)$ \\
Europe & 47 & $1,383,299,257$ & 79.2 & 0.102 & $10(95 \% \mathrm{Cl} 8.2-12.2)$ \\
America & 28 & $195,218,499$ & 87.9 & 0.110 & $19(95 \% \mathrm{Cl} 15-23.8)$ \\
Africa & 2 & 59,303 & 79.03 & - & $15.2(95 \% \mathrm{Cl} 6.9-30.2)$ \\
\hline
\end{tabular}

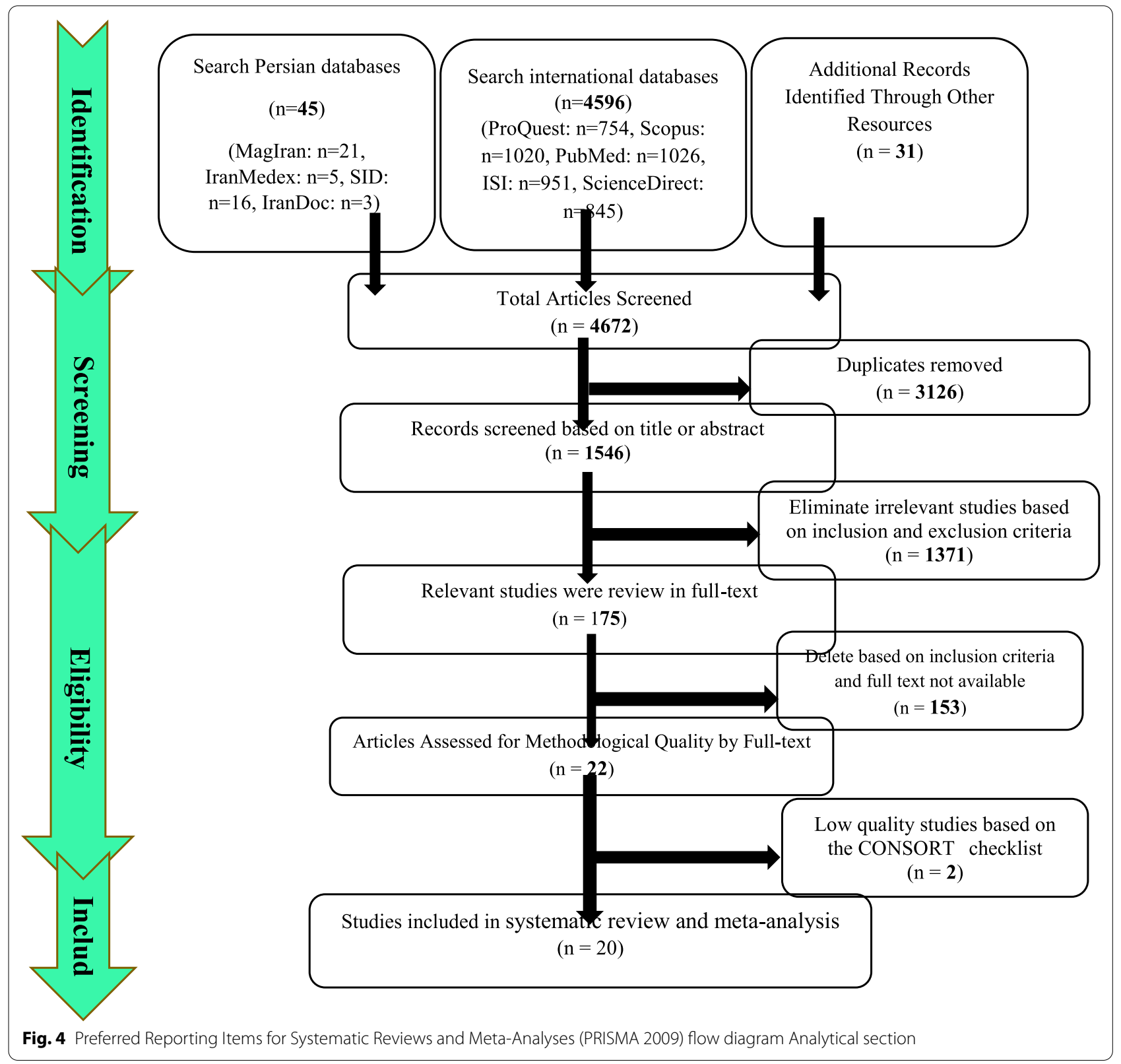




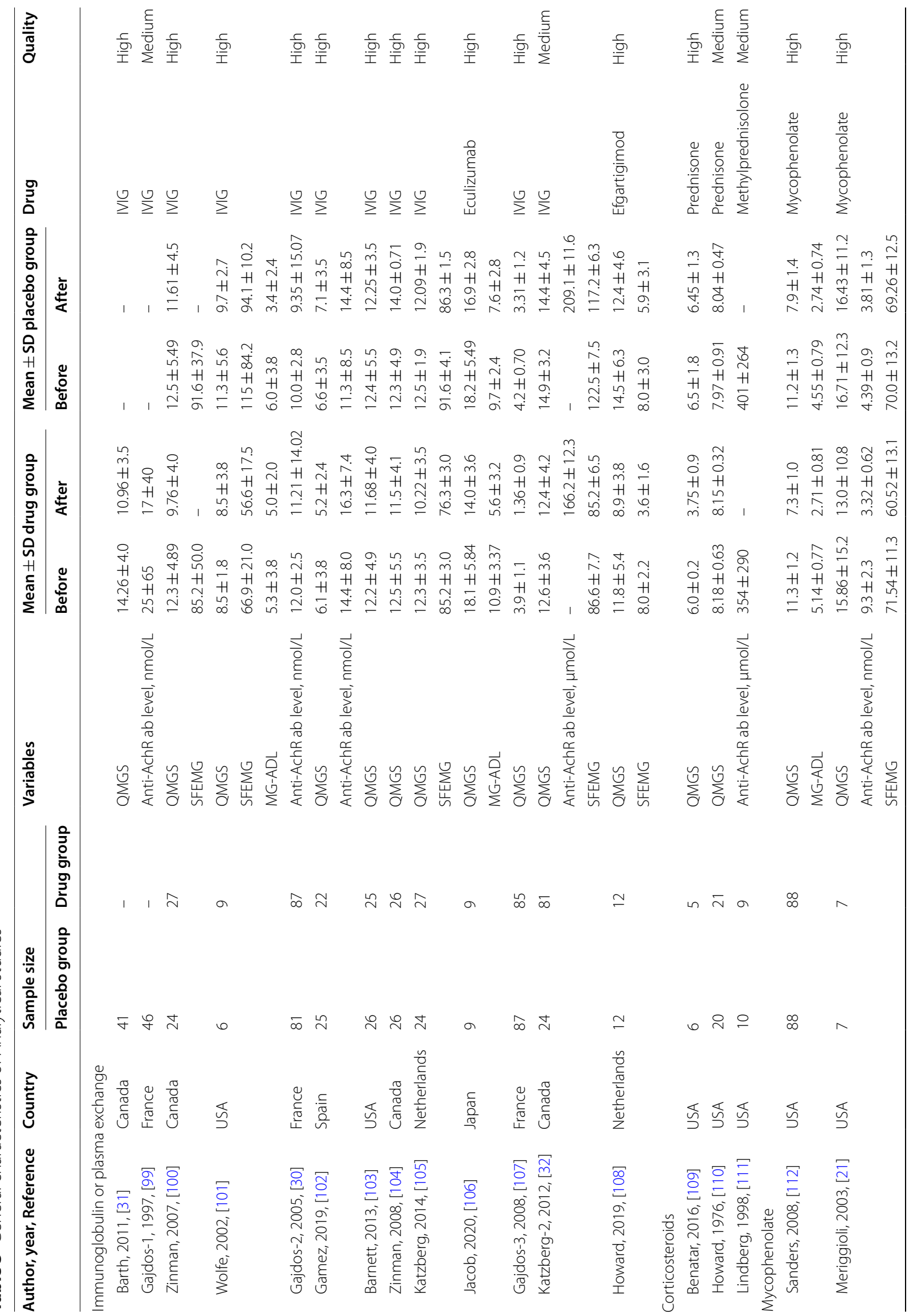




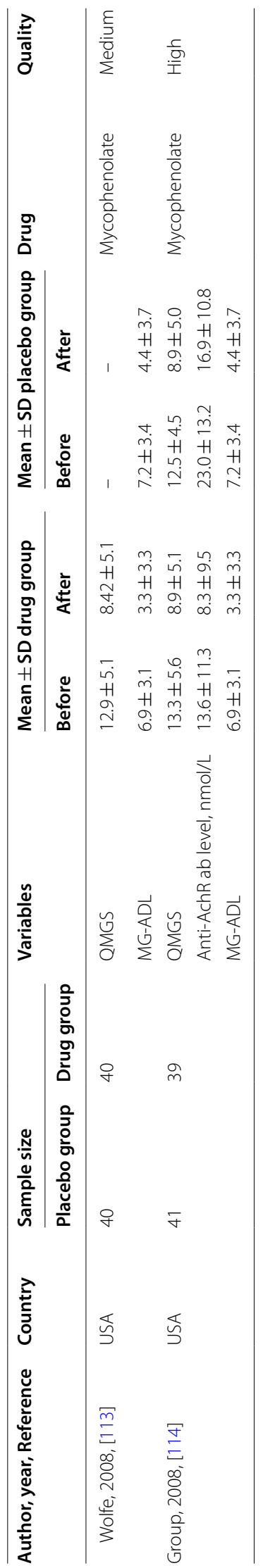


Table 4 Mean and standard deviation as well as the difference between the mean of drug evaluation indices before and after the intervention in the studied groups

\begin{tabular}{|c|c|c|c|c|c|c|}
\hline Drugs & Index & $1^{2}$ & $\begin{array}{l}\text { Begg and mazumdar } \\
\text { rank correlation test }\end{array}$ & $\begin{array}{l}\text { Mean } \pm \text { SD before } \\
\text { intervention (drug } \\
\text { group) }\end{array}$ & $\begin{array}{l}\text { Mean } \pm \text { SD after } \\
\text { intervention (drug } \\
\text { group) }\end{array}$ & $\begin{array}{l}\text { Mean difference and } \\
\text { standard deviation } \\
\text { before and after the } \\
\text { intervention }\end{array}$ \\
\hline \multirow[t]{4}{*}{ Mycophenolate } & MG-ADL & 85.3 & 1.000 & $5.9 \pm 0.87$ & $7.5 \pm 4.09$ & $1.4 \pm 0.9$ \\
\hline & SFEMG & 0 & - & $71.5 \pm 11.3$ & $60.5 \pm 13.1$ & $0.9 \pm 0.56$ \\
\hline & Anti-AChR antibodies & 79.1 & - & $11.1 \pm 2.1$ & $5.5 \pm 2.4$ & $1.9 \pm 1.5$ \\
\hline & QMGS & 67.9 & 0.734 & $12.3 \pm 0.71$ & $8.1 \pm 0.59$ & $1.4 \pm 0.77$ \\
\hline \multirow{4}{*}{$\begin{array}{l}\text { Immunoglobu- } \\
\text { lin or plasma } \\
\text { exchange }\end{array}$} & MG-ADL & 78.5 & 1.000 & $8.2 \pm 1.3$ & $4.4 \pm 0.84$ & $1.3 \pm 0.63$ \\
\hline & SFEMG & 43.5 & 1.000 & $82.2 \pm 1.43$ & $54.5 \pm 27.5$ & $1.5 \pm 0.73$ \\
\hline & Anti-AChR antibodies & 99.8 & 1.000 & $10.8 \pm 4.6$ & $52.7 \pm 34.1$ & $-2.006 \pm 0.78$ \\
\hline & QMGS & 98.6 & 0.391 & $11.2 \pm 1.6$ & $9.4 \pm 1.8$ & $0.62 \pm 0.28$ \\
\hline Corticosteroids & QMGS & 99.4 & - & $7.08 \pm 1.09$ & $5.9 \pm 2.2$ & $1.64 \pm 1.6$ \\
\hline
\end{tabular}

autoimmune attack of antibodies at the neuromuscular junction. Specific antibodies induce this autoimmune response against the acetylcholine receptor by blocking or binding to the receptor or postsynaptic membrane damage [119].

Based on the present meta-analysis results, there is a lot of heterogeneity between studies $\left(\mathrm{I}^{2}=99.8\right)$, so the stochastic effects model was used to combine the studies and the final result. Begg and Mazumdar rank correlation test Emission bias was not presented in studies for Anti-AChR antibodies index $(\mathrm{P}=1.000)$ (Table 4). As a result of the combination of studies, the mean score of Anti-AChR antibodies before the intervention in the drug group was $10.8 \pm 4.6$ and after the intervention was $52.7 \pm 34.1$ (Table 4). AChR antibodies were obtained before and after the intervention at $-2.006 \pm 78.7(\mathrm{P}<$ 0.01) (Fig. 7), indicating that Immunoglobulin or plasma exchange did not affect the Anti-AChR antibodies index. QMGS index.

\section{QMGS index}

Myasthenia Gravis Quantitative Score (QMGS) is a 13-item scale developed by Tindall et al. [120] and modified by Barohn et al. [121] to be used to determine the severity of myasthenia gravis. This scale measures ocular, bulbar, respiratory, and limb function and scores each finding from zero (no myasthenic findings) to 39 (maximum myasthenic defects) $[122,123]$.

Based on the present meta-analysis results, there is a lot of heterogeneity between studies $\left(\mathrm{I}^{2}=98.6\right)$, so the random-effects model was used to combine the studies and the final result. Begg and Mazumdar rank correlation test was not possible in the studies for the QMGS index $(\mathrm{P}=0.391)$ (Table 4).
As a result of the combination of studies, the mean score of QMGS indices before the intervention in the drug group was $11.2 \pm 1.6$ and after the intervention was $9 / 1 \pm 4 / 8$ (Table 4), as well as the difference between the mean of the QMGS index before and after the intervention. $0.62 \pm 0.28$ was obtained $(\mathrm{P}<0.01)$ (Fig. 8$)$, which indicates the positive effect of Immunoglobulin or plasma exchange on QMGS index reduction.

\section{Mycophenolate}

In total, 4 studies examined the effect of Mycophenolate on MG patients. 4 studies reviewed the QMGS index, 2 studies the Anti-AChR antibodies index, 1 study the SFEMG index and 3 studies the MG-ADL index.

\section{MG-ADL index}

Based on the results of the present meta-analysis studies, there is a lot of heterogeneity $\left(\mathrm{I}^{2}=85.3\right)$, so the stochastic effects model was used to combine the studies and the outcome. Begg and Mazumdar rank correlation test of publication bias was not possible in the studies for MGADL index $(\mathrm{P}=1.000)$ (Table 4$)$.

As a result of the combination of studies, the mean score of MG-ADL indices before the intervention in the drug group was $5.9 \pm 0.87$ and after the intervention was $7.4 \pm 5.09$ (Table 4), as well as the difference between the mean of the MGADL index before and after The intervention showed $1.4 \pm 0.9(\mathrm{P}<0.01)$ (Fig. 9) which indicates the positive effect of Mycophenolate on the reduction of MG-ADL index.

\section{SFEMG index}

Based on the present meta-analysis results between studies, according to a study, there was no heterogeneity $\left(\mathrm{I}^{2}=0\right)$, so the fixed effects model was used to combine 


\section{Meta Analysis}

\begin{tabular}{|c|c|c|c|c|c|c|c|c|c|c|c|}
\hline \multirow[t]{2}{*}{ Study name } & \multicolumn{7}{|c|}{ Statistics for each study } & \multicolumn{4}{|c|}{ Std diff in means and $95 \% \mathrm{Cl}$} \\
\hline & $\begin{array}{c}\text { Std diff } \\
\text { in means }\end{array}$ & $\begin{array}{l}\text { Standard } \\
\text { error }\end{array}$ & Variance & $\begin{array}{c}\text { Lower } \\
\text { limit }\end{array}$ & $\begin{array}{c}\text { Upper } \\
\text { limit }\end{array}$ & Z-Value & $\mathrm{p}$-Value & & & & \\
\hline Wolfe & 0.099 & 0.578 & 0.334 & $1.033-$ & 1.231 & 0.171 & 0.864 & & & & | \\
\hline Jacob & 1.613 & 0.543 & 0.294 & 0.549 & 2.676 & 2.972 & 0.003 & & & & \\
\hline \multirow{4}{*}{ Howard } & 2.287 & 0.525 & 0.276 & 1.258 & 3.317 & 4.357 & 0.000 & & & & \\
\hline & 1.351 & 0.636 & 0.405 & 0.104 & 2.598 & 2.124 & 0.034 & & & & \\
\hline & & & & & & & & -4.00 & -2.00 & 0.00 & 4.00 \\
\hline & & & & & & & & & Favours A & Favours B & \\
\hline
\end{tabular}

Meta Analysis

Fig. 5 Accumulation chart of studies included in the meta-analysis based on the difference between the mean MG-ADL index before and after the intervention for Immunoglobulin or plasma exchange

\section{Meta Analysis}

\begin{tabular}{|c|c|c|c|c|c|c|c|c|c|c|c|}
\hline \multirow{2}{*}{ Study name } & \multicolumn{7}{|c|}{ Statistics for each study } & \multicolumn{4}{|c|}{ Std diff in means and $95 \% \mathrm{Cl}$} \\
\hline & $\begin{array}{c}\text { Std diff } \\
\text { in means }\end{array}$ & $\begin{array}{l}\text { Standard } \\
\text { error }\end{array}$ & Variance & $\begin{array}{c}\text { Lower } \\
\text { limit }\end{array}$ & $\begin{array}{l}\text { Upper } \\
\text { limit }\end{array}$ & Z-Value & p-Value & & & & \\
\hline Zinman & 2.410 & 0.379 & 0.144 & 1.667 & 3.153 & 6.354 & 0.000 & & 1 & & \\
\hline Wolfe & 0.533 & 0.588 & 0.345 & $0.619-$ & 1.684 & 0.907 & 0.364 & & & & \\
\hline Katzberg-1 & 2.967 & 0.418 & 0.175 & 2.147 & 3.787 & 7.091 & 0.000 & & & & \\
\hline \multirow[t]{4}{*}{ Katzberg-2 } & 0.196 & 0.289 & 0.084 & $0.371-$ & 0.764 & 0.679 & 0.497 & & & & \\
\hline & 1.533 & 0.734 & 0.538 & 0.096 & 2.971 & 2.090 & 0.037 & & & & \\
\hline & & & & & & & & -4.00 & -2.00 & 2.00 & 4.00 \\
\hline & & & & & & & & & Favours A & Favours B & \\
\hline
\end{tabular}

Fig. 6 Accumulation chart of studies included in the meta-analysis based on the difference between the mean SFEMG index before and after the intervention for Immunoglobulin or plasma exchange

\section{Meta Analysis}

\begin{tabular}{|c|c|c|c|c|c|c|c|c|c|c|c|c|}
\hline \multirow[t]{2}{*}{ Study name } & \multicolumn{7}{|c|}{ Statistics for each study } & \multicolumn{5}{|c|}{ Std diff in means and $95 \% \mathrm{Cl}$} \\
\hline & $\begin{array}{l}\text { Std diff } \\
\text { in means }\end{array}$ & $\begin{array}{l}\text { Standard } \\
\text { error }\end{array}$ & Variance & $\begin{array}{c}\text { Lower } \\
\text { limit }\end{array}$ & $\begin{array}{c}\text { Upper } \\
\text { limit }\end{array}$ & Z-Value & p-Value & & & & & \\
\hline Gajdos-1 & 0.148 & 0.209 & 0.044 & $0.261-$ & 0.557 & 0.710 & 0.478 & | & & & | & \\
\hline Gajdos-2 & 0.078 & 0.157 & 0.025 & $0.230-$ & 0.387 & 0.499 & 0.618 & & & & & \\
\hline Gamez & $0.247-$ & 0.284 & 0.081 & $0.803-$ & 0.310 & $0.868-$ & 0.385 & & & & & \\
\hline \multirow[t]{4}{*}{ Katzberg-2 } & $19.109-$ & 1.972 & 3.887 & 22.973- 1 & $15.245-$ & $9.692-$ & 0.000 & $k$ & & & & \\
\hline & $2.006-$ & 0.787 & 0.620 & $3.549-$ & $0.464-$ & $2.549-$ & 0.011 & & & & & \\
\hline & & & & & & & & -4.00 & -2.00 & 0.00 & 2.00 & 4.00 \\
\hline & & & & & & & & & Favours A & & Favours B & \\
\hline
\end{tabular}

Meta Analysis

Fig. 7 Accumulation chart of studies included in the meta-analysis based on the mean difference of the anti-AChR antibodies index before and after the intervention for Immunoglobulin or plasma exchange 


\section{Meta Analysis}

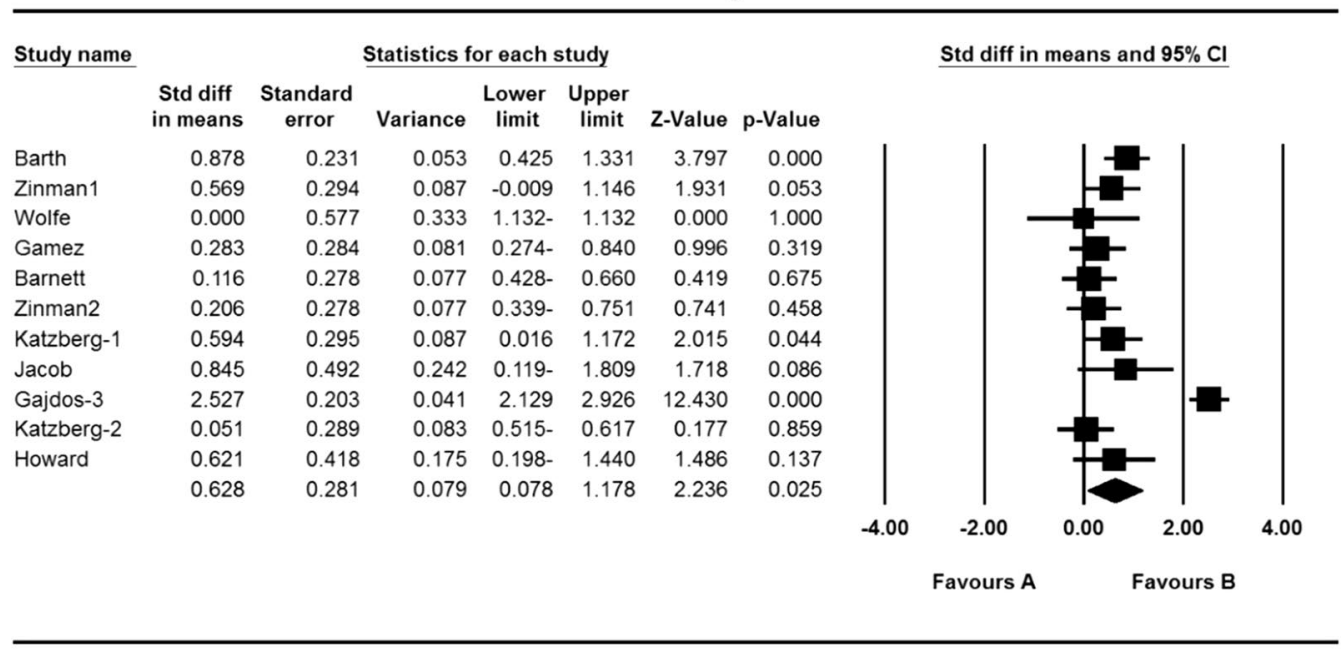

Meta Analysis

Fig. 8 Accumulation diagram of meta-analysis studies based on mean differences in mean QMGS before and after intervention for Immunoglobulin or plasma exchange

\begin{tabular}{|c|c|c|c|c|c|c|c|c|c|c|c|}
\hline \multicolumn{12}{|c|}{ Meta Analysis } \\
\hline Study name & \multicolumn{7}{|c|}{$\underline{\text { Statistics for each study }}$} & \multicolumn{4}{|c|}{ Std diff in means and $95 \% \mathrm{Cl}$} \\
\hline & $\begin{array}{c}\text { Std diff } \\
\text { in means }\end{array}$ & $\begin{array}{l}\text { Standard } \\
\text { error }\end{array}$ & Variance & $\begin{array}{l}\text { Lower } \\
\text { limit }\end{array}$ & $\begin{array}{c}\text { Upper } \\
\text { limit }\end{array}$ & Z-Value & $\mathrm{p}$-Value & & & & \\
\hline Sanders & 3.075 & 0.223 & 0.050 & 2.639 & 3.511 & 13.808 & 0.000 & & & & $F$ \\
\hline Wolfe & 0.049 & 0.224 & 0.050 & $0.390-$ & 0.487 & 0.218 & 0.827 & & & & \\
\hline Group & 1.124 & 0.238 & 0.056 & 0.659 & 1.590 & 4.731 & 0.000 & & & & \\
\hline & 1.416 & 0.904 & 0.817 & $0.355-$ & 3.188 & 1.567 & 0.000 & & & & \\
\hline & & & & & & & & -4.00 & -2.00 & 0.00 & 4.00 \\
\hline & & & & & & & & & Favours A & Favours B & \\
\hline
\end{tabular}

Fig. 9 Accumulation chart of studies entered for meta-analysis based on the difference between the mean MG-ADL index before and after intervention for Mycophenolate

the study and the final result. It was not possible to perform Begg and Mazumdar rank correlation test in the studies for the SFEMG index according to the study of only one research (Table 4).

As a result of the combination of studies, the mean score of SFEMG indices before the intervention in the drug group was $71.5 \pm 11.3$ and after the intervention was $60.5 \pm 13.1$ (Table 4), as well as the difference between the mean of the SFEMG index before and after the intervention. $0.9 \pm 0.56$ was obtained $(\mathrm{P}<0.01)$, indicating Mycophenolate's positive effect on SFEMG index reduction.

\section{Index of anti-AChR antibodies}

Based on the present meta-analysis results between studies, there is a lot of heterogeneity $\left(\mathrm{I}^{2}=79.1\right)$, so the stochastic effects model was used to combine the studies and the final result. It was not possible to perform Begg and Mazumdar rank correlation test, publication bias in studies for Anti AChR antibodies index due to review of only 2 studies (Table 4 ).

As a result of the combination of studies, the mean score of Anti-AChR antibodies before the intervention in the drug group was $11.1 \pm 2.1$ and after the intervention was $5.5 \pm 2.4$ (Table 4) and the difference between the 
mean of the anti-AChR index. Antibodies were obtained before and after the intervention $(1.9 \pm 1.5(\mathrm{P}<0.01)$ (Fig. 10)), which indicates the positive effect of Mycophenolate on the Anti-AChR antibodies index.

\section{QMGS index}

Based on the present meta-analysis results between studies, there is a lot of heterogeneity $\left(\mathrm{I}^{2}=67.9\right)$, so the stochastic effects model was used to combine the studies and the final results.

As a result of the combination of studies, the mean score of QMGS indices before the intervention in the drug group was $12.3 \pm 0.71$ and after the intervention was $8.0 \pm 0.59$ (Table 4). It was obtained $1.4 \pm 0.77(\mathrm{P}<0.01)$ (Fig. 11), indicating Mycophenolate's positive effect on QMGS index reduction.

\section{Corticosteroids}

In the study of studies in the field of corticosteroids, only the QMGS index could be examined. Based on this, 3 studies examined the effect of corticosteroids on MG patients. In the study of Benatar et al. [109], the QMGS index before the intervention in the placebo group was $6.5 \pm 1.8$ units and in the drug group was $6.0 \pm 0.2$ units, and after the intervention in the placebo group decreased by 0.05 units $(P>0.05)$. There was a significant decrease of 2.25 units $(\mathrm{P}<0.05)$ [109]. Also, in the study of Howard et al. [110] QMGS index before the intervention in the placebo group was $7.97 \pm 0.91$ units and in the drug group was $8.18 \pm 0.63$ units and after the intervention in the placebo group increased by 0.07 units $(\mathrm{P}>0.05)$ and in the drug group had a decrease of 0.03 units $(\mathrm{P}>$ 0.05) [110]. In the study of Lindberg et al. [111], The anti$\mathrm{AChR}$ antibodies index was reported before intervention

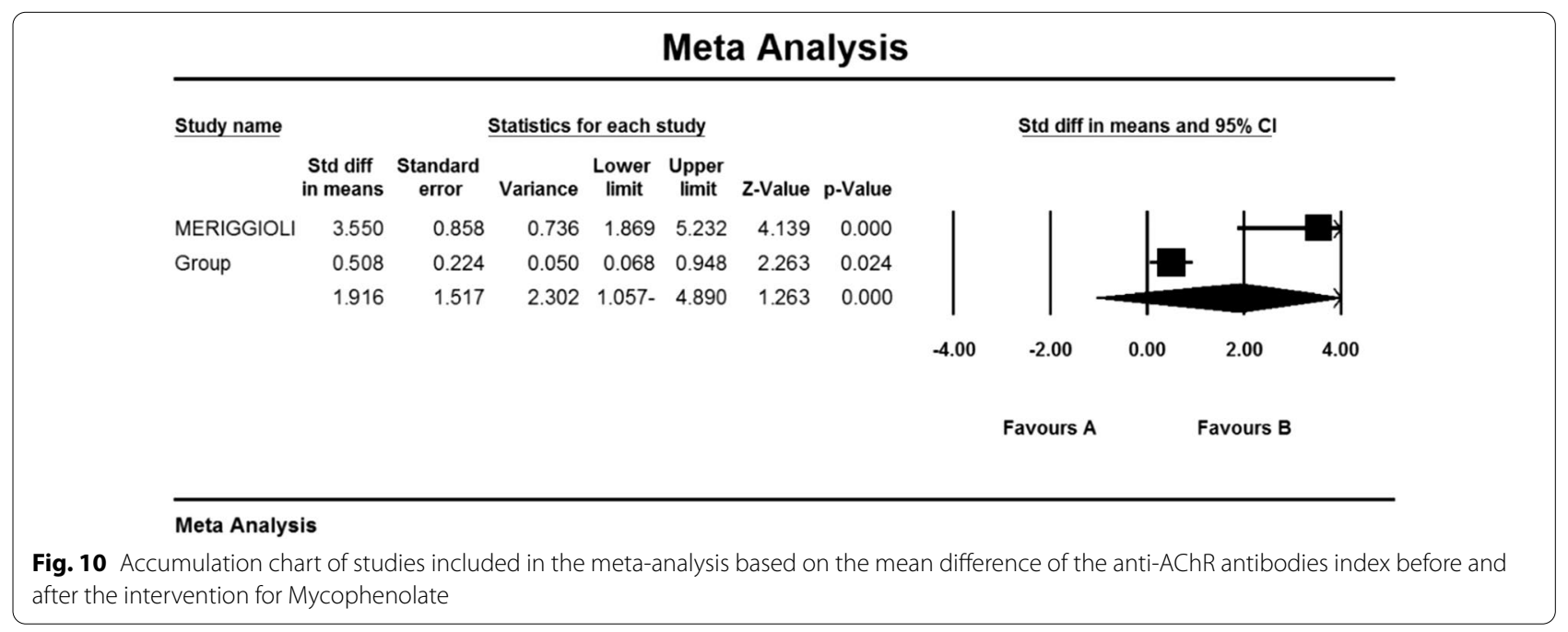

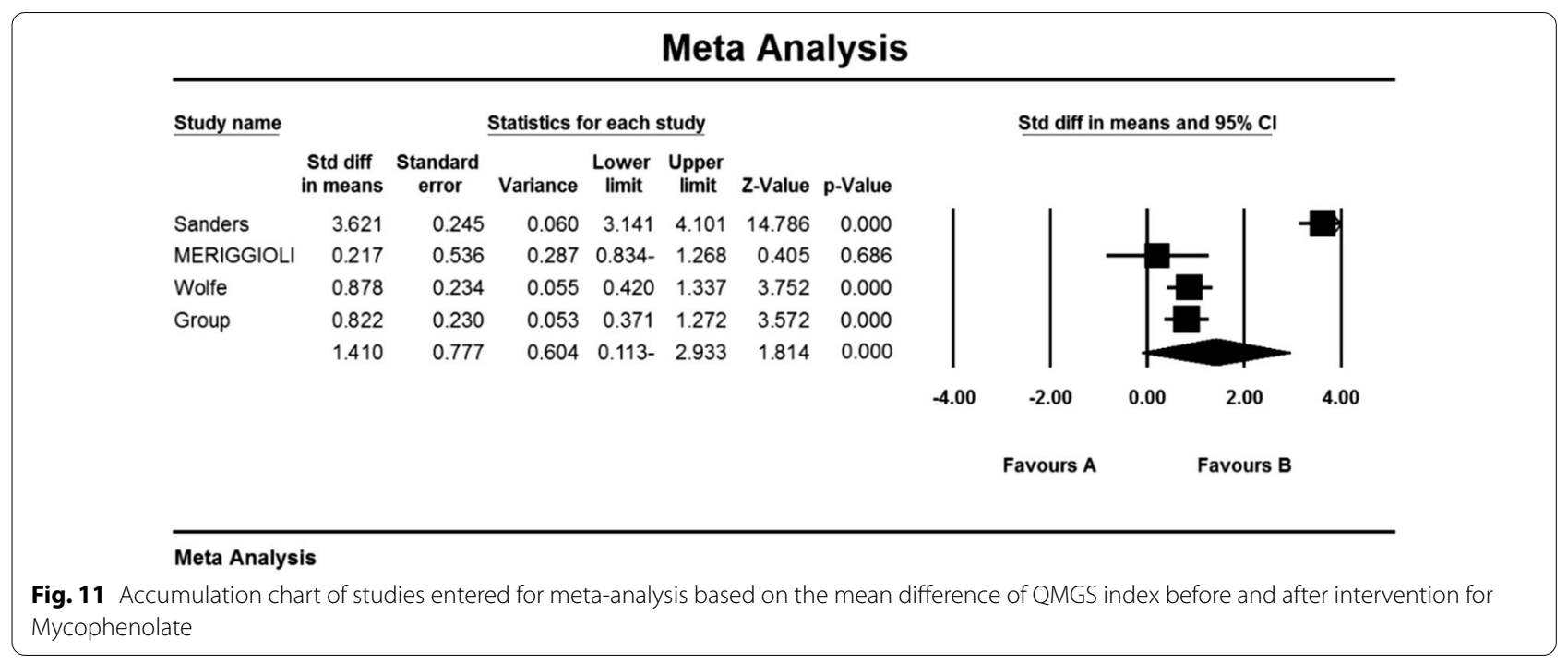


in the placebo group of $264 \pm 401(\mu \mathrm{mol} / \mathrm{L})$ and in the drug group of $354 \mathrm{~m} 290(\mu \mathrm{mol} / \mathrm{L})$ [111].

\section{QMGS index}

Based on the present meta-analysis results between studies, there is a lot of heterogeneity $\left(\mathrm{I}^{2}=99.4\right)$, so the stochastic effects model was used to combine the studies and the final result of the outcomes. According to the review of only two studies, it was not possible to use the Begg and Mazumdar rank correlation test for the QMGS index studies according to the review of only 2 studies (Table 4).

As a result of the combination of studies, the mean score of QMGS indices before the intervention in the drug group was 7.08091 .09 and after the intervention was 5.922 .2 (Table 4), as well as the difference between the mean scores of the QMGS index before and after the intervention. 1.6411 .6 was obtained (P.010.01) (Fig. 12), which indicates the positive effect of corticosteroids on reducing the QMGS index.

\section{Discussion}

Myasthenia gravis (MG) is the largest group of neuromuscular disorders caused by autoimmune antibodies against postsynaptic components of the voluntary muscle endplate [124-126]. Acetylcholine receptor antibodies (AChR), muscle-specific kinase (MuSK), and lipoproteinassociated protein (LRP4) have been well established as sensitive diagnostic markers and pathogens, in addition to antibodies in the classification of patients with Myasthenia gravis also play a key role [127].

Although the clinical features of MG can vary, increasing muscle weakness with continued skeletal muscle activity is one way to diagnose the disease [128]. Unlike ocular involvement, which is often asymmetric and involves several muscles, the pattern of muscle involvement in myasthenia gravis is usually symmetrical. Muscle weakness usually increases with exercise and frequent muscle use, and its intensity varies from day to day and fluctuates throughout the day [129].

In the current systematic review and meta-analysis study, the overall prevalence of MG in the world; 12.4 people per 100,000 population were obtained. Most prevalent in Salvado et al. [61]; 3463 per 100,000 population and the lowest prevalence Bettini et al. [58]; 0.006 people per 100,000 population reported.

Due to the different reports of MG prevalence in different parts of the world, a detailed study of the prevalence of this disease in different continents in order to pay more attention to planners and its consequences seemed necessary. Therefore, according to the subgroup analysis by different continents (Asia, Europe, Africa, and America), the highest prevalence of myasthenigraphy was reported in the Americas with 19 per 100,000 people and the lowest in continental Europe with 10 per 100,000 people.

Symptomatic, safe, and supportive approaches are very effective in treating myasthenia gravis, and treatment should be aimed at complete or almost complete drug recovery [130]. Most patients with myasthenia gravis to achieve therapeutic goals of full physical function or relatively high quality of life need immunosuppressive drugs. Immunosuppressive drugs are prescribed to all patients who respond only to symptomatic and supportive treatment [131].

Only the QMGS index could be assessed in studies of corticosteroids, which measures the severity of myasthenia gravis in 13 items [120]. The mean score of the QMGS index before and after the intervention in the drug group was 7.08091 .09 and 5.922 .2 , which indicates the positive effect of corticosteroid use on reducing the QMGS index improving myasthenia gravis.

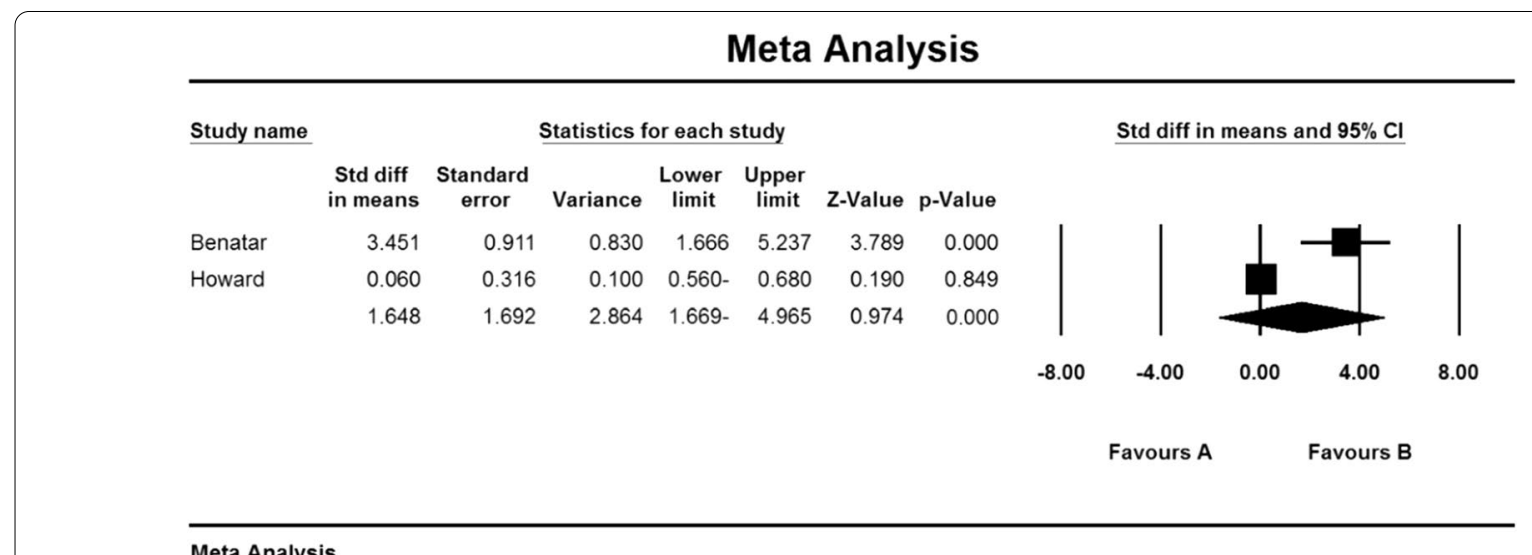

Fig. 12 Accumulation diagram of studies included in the meta-analysis based on the difference between the mean QMGS index before and after the intervention for Corticosteroids 
Oral corticosteroid therapy has been used since the 1950s with a dramatic improvement in approximately 70 to $80 \%$ of patients with myasthenia gravis $[132,133]$. The usefulness of oral steroids is determined by the occurrence of a wide range of dose and time-dependent side effects [134, 135]. Intermittent intravenous methylprednisolone (IVMP) is used to treat several autoimmune disorders, including MG, on the assumption that it is more effective and has fewer side effects than oral steroids [136]. IVMP is also effective in severe cases of MG [137].

Mycophenolate mofetil (MMF) is an immunosuppressive agent that is primarily used to prevent acute rejection of organ transplants [138] which have reported preliminary use of this drug in the treatment of myasthenia gravis [139].

Regarding the effectiveness of mycophenolate mofetil, the mean score of MG-ADL index before and after the intervention in the drug group was 5.9870 .87 and 7.409 5.09 , respectively. This scale assesses daily life activity in people with myasthenia gravis through 8 items [115]. The mean score of the SFEMG (single-strand electromyography) index, which is used to evaluate neuromuscular site abnormalities [117], was reported to be $71.5 \pm 11.3$ and 60.1355 .1 , respectively, before and after the intervention in the drug group. Also, the mean score of AntiAChR antibodies before and after the intervention in the drug group was $5 / 5 \pm 2 / 4$ and $11 / 2 \pm 1 / 1$. The mean score of QMGS indices before and after the intervention in the drug group was $12.3 \pm 0.71$ and $8.0 \pm 1.59$, which the results show the positive effect of using Mycophenolate on reducing the above 4 indicators and thus improving the treatment status of patients with MG.

Certain cure requires suppression or modulation of the immune system by intravenous immunoglobulin (IVIg) or plasma replacement (PLEX) [140]. Immune system modification is used when rapid recovery is required, such as exacerbated myasthenia gravis, power optimization before thymectomy, and patients who do not tolerate and respond adequately to immunosuppressive drugs $[100,141,142]$. In recent years, the administration of $2 \mathrm{~g} /$ $\mathrm{kg}$ intravenous IVIg immunoglobulin has been proven to treat moderate to severe myasthenia gravis and is continuously used to manage intensified MG [143].

Therapeutic plasmapheresis or plasma replacement (PLEX) is the first line of treatment in patients with myasthenia gravis with respiratory failure, inability to swallow, myasthenic crisis, or inadequate response to drug therapy $[144,145]$. In therapeutic plasmapheresis, plasma containing pathogenic antibodies is separated from the patient's blood and returned to other cells. Plasma replacement is prescribed five times in 10 to 14 days, through which and by repeating it, plasma levels of acetylcholine receptor antibody are reduced, and clinical improvement is achieved [119].

Due to the high prevalence of myasthenia gravis globally and its many negative consequences for individuals and society. Therefore, it seems useful to take measures to achieve better therapies or to use supportive therapies to reduce the symptoms of the disease. Common drug treatments in MG were evaluated to show the effectiveness of immunosuppressive drugs, including steroids and their modulators, including intravenous immunoglobulin (IVIg and plasma replacement) (PLEX). These studies can provide useful information to health care providers, enrich health care interventions, improve the quality of services, and ultimately improve the quality of life of these people. Therefore, it is suggested that physicians and the health care system give these drug classes more attention.

The application of nanotechnology is promising, given frustrating problems in therapeutic neurology [146]. Nanotechnology involves the manipulation of technological machinery at the atomic scale. For perspective, a nucleus is about $6 \mu \mathrm{m}$ across, a ribosome $20 \mathrm{~nm}$ in diameter, and a single strand of DNA $2 \mathrm{~nm}$ wide [146]. A typical human being is composed of 100 trillion cells. Nanotechnology has created novel devices for the treatment of various neurological diseases. Shrinkage of machinery, chip-based technologies, and the creation of unprecedented nanomaterials are contributing immensely to the reduction of morbidity [146, 147]

Considerable efforts are being focused on using nanoneuromedicine for disease treatment in the research laboratory. In the case of neurodegenerative diseases such as myasthenia gravis (MG), Alzheimer's disease (AD), Parkinson's disease (PD), amyotrophic lateral sclerosis (ALS), and multiple sclerosis (MS), nanomedicines have emerged as promising treatment options. Pathophysiological processes involving neuron inflammation and protein misfolding initiate a degeneration cycle within the cell. This can be thwarted using better drug targeting. Diagnosing and monitoring the end-effects of therapeutics is possible using nanoneurotechnology [146-148].

In addition to what has been said, the interest in graphene-based nanomaterials (GBNs) application in nanomedicine, particularly neurology, steadily increased in the last decades. GBNs peculiar physical-chemical properties allow the design of innovative therapeutic tools to manipulate biological structures with subcellular resolution $[148,149]$. Based on the study, it can also be said that to develop effective antioxidant therapies the best strategy may be to create new nanoscale drug delivery systems [150]. 


\section{Limitations}

Among the limitations of this study, it can be asserted that some samples were not based on random selection. Also, non-uniform reporting of articles, inconsistent implementation method, non-copying and unavailability of the full text of articles presented at the conference can be mentioned as other limitations.

\section{Suggestion for future works}

The meta-analysis results enable the overall prevalence to be presented to the policy-maker and thus manage the cost, time and future diagnostic and treatment decisions commensurate with the overall prevalence. A systematic review also reveals drugs effective in treating myasthenia gravis, which can guide physicians and encourage the researcher to conduct future clinical trial studies and a network meta-analysis to determine therapeutic supplements for the disease.

\section{Conclusion}

The results of systematic review of drug evaluation in patients with myasthenia gravis showed that Mycophenolate and Immunoglobulin or plasma exchange drugs have positive effects in the treatment of MG. It also represents the positive effect of immunoglobulin or plasma exchange on reducing SFEMG index and QMGS index and the positive effect of Mycophenolate on reducing MG-ADL index, SFEMG and Anti-AChR antibodies index. In addition to what was mentioned, based on a meta-analysis of the random-effect model, the overall prevalence of MG in the world is 12.4 people per 100,000 populations, which indicates the urgent need for the attention of officials and specialists to this disease for prevention and treatment.

\section{Abbreviations \\ SID: Scientific Information Database; MESH: Medical Subject Headings; WoS: Web of science; PRISMA: Preferred Reporting Items for Systematic Reviews and Meta-Analysis; CONSORT: Consolidated Standards of Reporting Trials; STROBE: Strengthening the reporting of observational studies in epidemiology for a cross-sectional study; MG: Myasthenia Gravis; Anti-AChR ab: Anti-acetylcholine receptor antibodies; IVIG: Intravenous immunoglobulin; QMGS: Quantitative Myasthenia Gravis Score; MG-ADL: Myasthenia Gravis Activities of Daily Living; SFEMG: Single-fibre electromyography.}

\section{Acknowledgements}

This study results from research project No. 4000257 approved by the Student Research Committee of Kermanshah University of Medical Sciences. We would like to thank the esteemed officials of the centre for the financial affords of this study.

\section{Authors' contributions}

$\mathrm{NS}, \mathrm{BF}$, and MK contributed to the design and MM statistical analysis and participated in most study steps. MM and BF, and MK prepared the manuscript. YB and RF and MM and BF and PM and SS helped design and interpret the study. All authors read and approved the content of the manuscript.

Funding

By Deputy for Research and Technology, Kermanshah University of Medical Sciences (IR) (4000257). This deputy has no role in the study process.

\section{Availability of data and materials}

Datasets are available through the corresponding author upon reasonable request.

\section{Declarations}

\section{Ethics approval and consent to participate}

Ethics approval was received from the deputy of the research and technology ethics committee, Kermanshah University of Medical Sciences (IR.KUMS. REC.1400.116)

\section{Consent for publication}

Not applicable.

\section{Competing interests}

The authors declare that they have no conflict of interest.

\section{Author details}

${ }^{1}$ Department of Biostatistics, School of Health, Kermanshah University of Medical Sciences, Kermanshah, Iran. ${ }^{2}$ Student Research Committee, Kermanshah University of Medical Sciences, Kermanshah, Iran. ${ }^{3}$ Department of Translation Studies, Faculty of Literature, Istanbul University, Istanbul, Turkey. ${ }^{4}$ Department of Neurosurgery, School of Medicine, Kermanshah University of Medical Sciences, Kermanshah, Iran. ${ }^{5}$ Department of Neurology, School of Medicine, Kermanshah University of Medical Sciences, Kermanshah, Iran. ${ }^{6}$ Department of Biology, Faculty of Science, University Putra Malaysia, Serdang, Selangor, Malaysia. ${ }^{7}$ Cellular and Molecular Research Center, Gerash University of Medical Sciences, Gerash, Iran.

Received: 27 October 2021 Accepted: 6 December 2021

Published online: 20 December 2021

\section{References}

1. Sanders DB, Wolfe Gl, Benatar M, Evoli A, Gilhus NE, Illa I, et al. International consensus guidance for management of myasthenia gravis: executive summary. Neurology. 2016;87(4):419-25.

2. Gilhus NE, Verschuuren JJ. Myasthenia gravis: subgroup classification and therapeutic strategies. Lancet Neurol. 2015;14(10):1023-36.

3. Shield TW, editor. General thoracic surgery, 7th ed. Philadelphia: Wolters Kluwer Health/Lippincott Williams \& Wilkins; 2010. pp. 2323-64.

4. Conti-Fine BM, Milani M, Kaminski HJ. Myasthenia gravis: past, present, and future. J Clin Invest. 2006;1 16(11):2843-54.

5. Benatar M. A systematic review of diagnostic studies in myasthenia gravis. Neuromuscul Disord. 2006;16(7):459-67.

6. Berrih-Aknin S, Frenkian-Cuvelier M, Eymard B. Diagnostic and clinical classification of autoimmune myasthenia gravis. J Autoimmun. 2014;48-49:143-8.

7. Meriggioli MN, Sanders DB. Autoimmune myasthenia gravis: emerging clinical and biological heterogeneity. Lancet Neurol. 2009;8(5):475-90.

8. Hoch W, McConville J, Helms S, Newsom-Davis J, Melms A, Vincent A. Auto-antibodies to the receptor tyrosine kinase MuSK in patients with myasthenia gravis without acetylcholine receptor antibodies. Nat Med. 2001;7(3):365-8.

9. Higuchi O, Hamuro J, Motomura M, Yamanashi Y. Autoantibodies to low-density lipoprotein receptor-related protein 4 in myasthenia gravis. Ann Neurol. 2011;69(2):418-22.

10. Kerty E, Elsais A, Argov Z, Evoli A, Gilhus NE. EFNS/ENS guidelines for the treatment of ocular myasthenia. Eur J Neurol. 2014;21:687-93.

11. Heldal AT, Owe JF, Gilhus NE, Romi F. Seropositive myasthenia gravis: a nationwide epidemiologic study. Neurology. 2009;73:150-1. 
12. Wang L, Zhang S, Xi J, Li W, Zhou L, Lu J, et al. Efficacy and safety of tacrolimus for myasthenia gravis: a systematic review and meta-analysis. J Neurol. 2017;264(11):2191-200.

13. Carr AS, Cardwell CR, McCarron PO, McConville J. A systematic review of population-based epidemiological studies in myasthenia Gra-vis. BMC Neurol. 2010;10:46.

14. MacDonald BK, Cockerell OC, Sander JW, Shorvon SD. The incidence and lifetime prevalence of neurological disorders in a prospective community-based study in the UK. Brain J Neurol. 2000;123(Pt 4):665-76.

15. Breiner A, Widdifield J, Katzberg HD, Barnett C, Bril V, Tu K. Epidemiology of myasthenia gravis in Ontario, Canada. Neuromuscular Disord. 2016;26(1):41-6.

16. Kurtzke JF. Epidemiology of myasthenia gravis. Adv Neurol. 1978;19:545-66.

17. Eaton WW, Rose NR, Kalaydjian A, Pedersen MG, Mortensen PB. Epidemiology of autoimmune diseases in Denmark. J Autoimmun. 2007;29(1):1-9.

18. Mh H, Ms F. The clinical outcome of thymectomy in myasthenia gravis. Tehran Univ Med J TUMS Publications. 2009;66(11):821-5.

19. Chen S, Xu M-B, Zhou X-L, Rong P-Q, Jin T-Y, Zheng G-Q. Chinese herbal medicine for myasthenia gravis: a systematic review and meta-analysis. Front Pharmacol. 2018;9:969.

20. Li H-F, Xie Y, Yue Y-X. Myasthenia gravis: subgroup classifications. Lancet Neurol. 2016:15(4):355-6.

21. Meriggioli MN, Rowin J, Richman JG, Leurgans S. Mycophenolate mofetil for myasthenia gravis: a double-blind, placebo-controlled study. Ann NY Acad Sci. 2003;998:494-9.

22. Sieb J. Myasthenia gravis: an update for the clinician. Clin Exp Immunol. 2014;175(3):408-18.

23. De Feo LG, Schottlender J, Martelli NA, Molfino NA. Use of intravenous pulsed cyclophosphamide in severe, generalized myasthenia gravies. Muscle Nerve. 2002;26(1):31-6.

24. García-Carrasco M, Escárcega RO, Fuentes-Alexandro S, Riebeling C, Cervera R. Therapeutic options in autoimmune myasthenia gravis. Autoimmun Rev. 2007:6(6):373-8.

25. Ciafaloni E. Mycophenolate mofetil and myasthenia gravis. Lupus. 2005;14(3-suppl):46-9.

26. Skeie GO, Apostolski S, Evoli A, et al. Guidelines for the treatment of autoimmune neuromuscular transmission disorders. Eur J Neurol. 2006;13(7):691-9.

27. Rozsa C, Lovas G, Fornadi L, Szabo G, Komoly S. Safety of long-term combined immunosuppressive treatment in myasthenia gravis: analysis of adverse effects of 163 patients. Eur J Neurol. 2006;13(9):947-52.

28. Drachman DB, Adams RN, Hu R, et al. Rebooting the immune system with high-dose cyclophosphamide for the treatment of refractory myasthenia gravis. Ann N Y Acad Sci. 2008;1132:305-14.

29. Suh J, Goldstein JM, Nowak RJ. Clinical characteristics of refractory myasthenia gravis patients. Yale J Biol Med. 2013;86(2):255-60

30. Gajdos P, Tranchant C, Clair B, Bolgert F, Eymard B, Stojkovic T, et al. Treatment of myasthenia gravis exacerbation with intravenous immunoglobulin: a randomized double-blind clinical trial. Arch Neurol. 2005;62(11):1689-93.

31. Barth D, Nabavi Nouri M, Ng E, Nwe P, Bril V. Comparison of IVIgand PLEX in patients with myasthenia gravis. Neurology. 2011;76(23):2017-23.

32. Katzberg HD, Barnett C, Bril V. Predictors of response to immunomodulation in patients with myasthenia gravis. Muscle Nerve. 2012:45(5):648-52.

33. Nagayasu T, Yamayoshi T, Matsumoto K, Ide N, Hasazumi S, Nomura $M$, et al. Beneficial effects of plasmapheresis before thymectomy on the outcome in myasthenia gravis. Jpn J Thorac Cardiovasc Surg. 2005:53(1):2-7.

34. Guptill JT, Sharma BK, Marano A, Soucy A, Krueger A, Sanders DB. The estimated cost of treating myasthenia gravis in an insured US population. Muscle Nerve. 2012;45(3):363-6.

35. Henderson LK, Craig JC, Willis NS, Tovey D, Webster AC. How to write a cochrane systematic review. Nephrology. 2010;15(6):617-24.

36. Ramke J, Palagyi A, Jordan V, Petkovic J, Gilbert CE. Using the STROBE statement to assess reporting in blindness prevalence surveys in low and middle-income countries. Plos One. 2017;12(5):e0176178.
37. Schulz KF, Altman DG, Moher D, Group C. CONSORT 2010 statement: updated guidelines for reporting parallel group randomized trials. Trials. 2010;11(1):32

38. Murai H, Yamashita N, Watanabe M, Nomura Y, Motomura M, Yoshikawa $\mathrm{H}$, et al. Characteristics of myasthenia gravis according to onset-age: Japanese nationwide survey. J Neurol Sci. 2011;305(1-2):97-102.

39. Nemet AY, Kaiserman I, Mimouni M, Segal O, Vinker S. High prevalence of myasthenia gravis among rural adult populations. J Clin Neuromuscul Dis. 2014;16(2):47-50.

40. Park S-Y, Lee JY, Lim NG, Hong Y-H. Incidence and prevalence of myasthenia gravis in Korea: a population-based study using the National Health Insurance claims database. J Clin Neurol. 2016;12(3):340-4.

41. Lee HS, Lee HS, Shin HY, Choi Y-C, Kim SM. The epidemiology of myasthenia gravis in Korea. Yonsei Med J. 2016;57(2):419-25.

42. Okinaka S, Reese HH, Katsuki S, et al. The prevalence of multiple sclerosis and other neurological diseases in Japan. Acta Neurologica Scandinavica. 1966;47(Suppl 19):68-76.

43. Araki S, Uchino M, Yoshida O. Epidemiologic study of multiple sclerosis, myasthenia gravis and polymyositis in the city of Kumamoto, Japan. Clin Neurol. 1983:23:838-41.

44. Kondo K, Takasu T, Ahmed A. Neurological diseases in Karachi, Pakistan-elevated occurrence of subacute sclerosing panencephalitis. Neuroepidemiology. 1988;7:66-80.

45. Yu YL, Hawkins BR, Ip MS, Wong V, Woo E. Myasthenia gravis in Hong Kong Chinese: epidemiology and adult disease. Acta Neurol Scand. 1992:86(2):113-9.

46. Zieda A, Ravina K, Glazere I, Pelcere L, Naudina M, Liepina L, et al. A nationwide epidemiological study of myasthenia gravis in Latvia. Eur J Neurol. 2018;25(3):519-26.

47. Lavrnic D, Basta I, Rakocevic-Stojanovic V, Stevic Z, Peric S, Nikolic A, et al. Epidemiological study of adult-onset myasthenia gravis in the area of Belgrade (Serbia) in the period 1979-2008. Neuroepidemiology. 2013;40(3):190-4.

48. Tola M, Granieri E, Paolino E, Caniatti L, Quatrale R, Mazzanti B, et al. Epidemiological study of myasthenia gravis in the province of Ferrara, Italy. J Neurol. 1989;236(7):388-90.

49. Montomoli C, Citterio A, Piccolo G, Cioccale R, Ferretti W, Fratti C, et al. Epidemiology and geographical variation of myasthenia gravis in the province of Pavia, Italy. Neuroepidemiology. 2012;38(2):100-5.

50. Cetin H, Fülöp G, Zach H, Auff E, Zimprich F. Epidemiology of myasthenia gravis in Austria: rising prevalence in an ageing society. Wien Klin Wochenschr. 2012;124(21-22):763-8.

51. Storm-Mathisen A. Epidemiology of myasthenia gravis in Norway. Acta Neurol Scand. 1984;70(4):274-84.

52. Westerberg E, Punga AR. Epidemiology of Myasthenia gravis in Sweden 2006-2016. Brain Behav. 2020:10:e01819.

53. Kalb B, Matell G, Pirskanen R, Lambe M. Epidemiology of myasthenia gravis: a population-based study in Stockholm, Sweden. Neuroepidemiology. 2002;21(5):221-5.

54. Aiello I, Pastorino M, Sotgiu S, Pirastru M, Sau G, Sanna G, et al. Epidemiology of myasthenia gravis in northwestern Sardinia. Neuroepidemiology. 1997;16(4):199-206.

55. Guidetti D, Sabadini R, Cavalletti S, Lodesani M, Mantegazza R, Solime FCV. Epidemiological study of myasthenia gravis in the province of Reggio Emilia, Italy. Eur J Epidemiol. 1998;14(4):381-7.

56. Foldvari A, Kovacs N, Sipos V, Merth G, Vincze F, Szucs M, et al. Estimation of incidence, prevalence, and age-at-diagnosis of myasthenia gravis among adults by hospital discharge records. Wien Klin Wochenschr. 2015;127(11-12):459-64.

57. Zivadinov R, Jurjevic A, Willheim K, Cazzato G, Zorzon M. Incidence and prevalence of myasthenia gravis in the County of the Coast and Gorski kotar, Croatia, 1976 through 19961. Neuroepidemiology. 1998;17(5):265-72.

58. Bettini M, Chaves M, Cristiano E, Pagotto V, Perez L, Giunta D, et al. Incidence of autoimmune myasthenia gravis in a health maintenance organization in Buenos Aires, Argentina. Neuroepidemiology. 2017;48(3-4):119-23.

59. Andersen J, Heldal A, Engeland A, Gilhus N. Myasthenia gravis epidemiology in a national cohort; combining multiple disease registries. Acta Neurol Scand. 2014;129:26-31. 
60. Aragones J, Altimiras J, Roura P, Alonso F, Bufill E, Munmany A, et al. Prevalence of myasthenia gravis in the Catalan county of Osona. Neurología (English Edition). 2017;32(1):1-5.

61. Salvado M, Canela M, Ponseti JM, Lorenzo L, Garcia C, Cazorla S, et al. Study of the prevalence of familial autoimmune myasthenia gravis in a Spanish cohort. J Neurol Sci. 2016;360:110-4.

62. Christensen P, Jensen T, Tsiropoulos I, Søsrensen T, Kjser M, HøjerPedersen $\mathrm{E}$, et al. Incidence and prevalence of myasthenia gravis in western Denmark: 1975 to 1989. Neurology. 1993;43(9):1779.

63. Robertson N, Deans J, Compston D. Myasthenia gravis: a populationbased epidemiological study in Cambridgeshire, England. J Neurol Neurosurg Psychiatry. 1998;65(4):492-6.

64. Garland H, Clark ANG. Myasthenia gravis, a personal study of 60 cases. BMJ. 1956;1:1259-62.

65. Pennington GW, Wilson A. Incidence of myasthenia gravis in the Merseyside conurbation. In: Veits HR, editor. Myasthenia Gravis. Proceedings of the second international symposium. Springfield IL: Charles C Thomas; 1961; pp. 337-45.

66. Gudmundsson KR. The prevalence of some neurological diseases in Iceland. Acta Neurol Scand. 1968:44:55-69.

67. Oosterhuis: Epidemiologie dei myasthenie in Amsterdam. Neurologie Deutsche Jesells. 1977; 103-108.

68. Hokkanen E. Epidemiology of myasthenia gravis in Finland. J Neurol Sci. 1969:9:463-78.

69. Giagheddu M, Puggioni G, Sanna G, et al. Epidemiological study of myasthenia gravis in Sardinia Italy (1958-1986). Acta Neurol Scand. 1989:79:326-33.

70. D'Alessamdro R, Granieri E, Benassi G, et al. Comparative study on the prevalence of myasthenia gravis in the provinces of Bologna and Ferrera Italy. Acta Neurol Scand. 1991;83(2):83-8.

71. Sorensen TT, Holm EB. Myasthenia gravis in the county of Viborg, Denmark. Eur Neurol. 1989;29:177-9.

72. Somnier FE, Keiding N, Paulson OB. Epidemiology of myasthenia gravis in Denmark: a longitudinal and comprehensive population survey. Arch Neurol. 1991;48(7):733-9.

73. Christensen PB, Jensen TS, Tsiropoulos I, et al. Mortality and survival in myasthenia gravis: a Danish population-based study. JNNP. 1998:64(1):78-83.

74. Ferrari G, Lovaste MG. Epidemiology of myasthenia gravis in the province of Trento (northern Italy). Neuroepidemiology. 1992;11(3):135-42.

75. Krivopusk ME. Clinico-epidemiological aspects of hereditary neuromuscular diseases in the Krasnodar territory. Zhurnal Nevropatologii I Psikhiatrii Imeni SS Korsakova. 1991;91(9):3-5.

76. Lavrnic D, Jarebinski M, Rakocevic-Stojanovic V, et al. Epidemiological and clinical characteristics of myasthenia gravis in Belgrade, Yugoslavia (1983-1992). Acta Neurologica Scandanavica. 1999;100(3):168-74.

77. Kyriallis K, Hristova A, Middleton I. What is the real epidemiology of myasthenia gravis? Neurology. 1995; A351.

78. Holtsema H, Mourik J, Rico RE, et al. Myasthenia gravis on the Dutch Antilles: an epidemiological study. Clin Neurol Neurosurg. 2000;102(4):195-8.

79. Villagra-Cocco A, Villagra-Cocco P. Prevalence of myasthenia gravis on the island of La Palma. Revista Neurol. 1997;25(148):2068-9.

80. Oopik M, Kaasik AE, Jakobson J. A population-based epidemiological study of myasthenia gravis in Estonia. JNNP. 2003;74(12):1638-43.

81. Wirtz PW, Nijnuis MG, Sotodeh M, et al. The epidemiology of myasthenia gravis, Lambert Eaton myasthenic syndrome and their associated tumours in the northern part of Southern Holland. J Neurol. 2003;250(6):698-701.

82. Kotov SV, Neretin VI, Agafonov BV, Sidorova OP. Population-based study of Myasthenia in Moscow region. Zhurnal Nevrologii I Psikhiatrii Imeni SS Korsakova. 2006;106(5):52-5.

83. Somnier FE. Increasing incidence of late-onset anti-AChR antibodyseropositive myasthenia gravis. Neurology. 2005;65:928-30.

84. Poulas K, Tsibri E, Kokla A, et al. Epidemiology of seropositive myasthenia gravis in Greece. JNNP. 2001;71(3):352-6.

85. Niks EH, Kuks JB, Verschuuren JJ, et al. Epidemiology of myasthenia gravis with anti-muscle specific kinase antibodies in The Netherlands. JNNP. 2007;78(4):417-8.
86. Tsiamalos P, Kordas G, Kokla A, et al. Epidemiological and immunological profile of muscle-specific kinase myasthenia gravis in Greece. Eu J Neurol. 2009;16(8):925-30.

87. Maharaj J, Bahadursingh S, Ramcharan K. Myasthenia gravis in South Trinidad. West Indian Med J. 2013;62(6):510-4.

88. Gordon B, Noone J, Van Doren B, Zacherle E, Blanchette C. Prevalence and cost of myasthenia gravis in the medicare beneficiary sample. Value Health. 2015;18(7):A661.

89. Phillips LH, Torner JC, Anderson MS, Cox GM. The epidemiology of myasthenia gravis in central and western Virginia. Neurology. 1992;42(10):1888.

90. Kurland LT. Descriptive epidemiology if selected neurologic and myopathic disorders with particular reference to a survey in Rochester, Minnesota. J Chronic Disorders. 1958;8(4):378.

91. Alter M, Rhett-Talbert $\mathrm{O}$, Kurland LT. Myasthenia gravis in a southern community. Arch Neurol. 1960;3:65-9.

92. Kvirkveliia NB. Clinico-epidemiologic aspects of Myasthenia in the Georgian SSR. Zh Nevropatol Psikhiatr Im S S Korsakova. 1986;86(3):327-30.

93. Cisernos AD, Luis RS, Leon R, Carrera PL. Some epidemiological aspects of myasthenia gravis in Cuba. Revista de Neurol. 1996;24(128):435-9.

94. Sanchez JL, Uribe CS, Franco AF, Jimeniz ME, Arcos-Burgos OM, Palacio LG. Prevalence of myasthenia gravis in Antioquia, Colombia. Revista de Neurologia. 2002;34(11):1010-2.

95. Deffeminis Rospide HA, Petra de Mirabel M, Piazza de Silva N, et al. Estudio epidemiologico de la miastenia en el Uruguay. Acta Neurol Latinoamer 1975; 53-65.

96. Khedr EM, Fawi G, Abbas MA-A, El-Fetoh AN, Zaki AF, Gamea A, et al. Prevalence of neuromuscular disorders in Qena governorate/Egypt: a population-based survey. Neurol Res. 2016; 38(12):1056-63

97. El-Tallawy HN, Khedr EM, Qayed MH, Helliwell TR, Kamel NF. Epidemiological study of neuromuscular disorders in Assuit, Egypt. Neuroepidemiology. 2005;25(4):205-11.

98. Gattellari M, Goumas C, Worthington J. A national epidemiological study of myasthenia gravis in Australia. Eur J Neurol. 2012;19(11):1413-20.

99. Gajdos P, Chevret S, Clair B, Tranchant C, Chastang C, Group MGCS. Clinical trial of plasma exchange and high-dose intravenous immunoglobulin in myasthenia gravis. Ann Neurol. 1997:41 (6):789-96.

100. Zinman L, Ng E, Bril V. IV immunoglobulin in patients with myasthenia gravis: a randomized controlled trial. Neurology. 2007;68(11):837-41.

101. Wolfe GI, Barohn RJ, Foster BM, Jackson CE, Kissel JT, Day JW, Thornton CA, Nations SP, Bryan WW, Amato AA. Randomized, controlled trial of intravenous immunoglobulin in myasthenia gravis. Muscle Nerve. 2002;26(4):549-52.

102. Gamez J, Salvadó M, Carmona F, de Nadal M, Romero L, Ruiz D, Jáuregui A, Martínez O, Pérez J, Suñé P. Intravenous immunoglobulin to prevent myasthenic crisis after thymectomy and other procedures can be omitted in patients with well-controlled myasthenia gravis. Ther Adv Neurol Disord. 2019:12:1756286419864497.

103. Barnett TC, Bril V, Davis AM. Performance of individual items of the quantitative myasthenia gravis score. Neuromuscul Disord. 2013;23(5):413-7.

104. Zinman L, Bril V. IVIG treatment for myasthenia gravis: effectiveness, limitations, and novel therapeutic strategies. Ann NY Acad Sci. 2008;1132(1):264-70

105. Katzberg HD, Barnett C, Merkies IS, Bril V. Minimal clinically important difference in myasthenia gravis: outcomes from a randomized trial. Muscle Nerve. 2014:49(5):661-5.

106. Jacob S, Murai H, Utsugisawa K, Nowak RJ, Wiendl H, Fujita KP, O'Brien F, Howard JF Jr. Response to eculizumab in patients with myasthenia gravis recently treated with chronic IVIg: a subgroup analysis of REGAIN and its open-label extension study. Ther Adv Neurol Disord. 2020;13:1756286420911784.

107. Gajdos $\mathrm{P}, \mathrm{Chevret} \mathrm{S}$. Treatment of myasthenia gravis acute exacerbations with intravenous immunoglobulin. Ann NY Acad Sci. 2008;1132(1):271-5

108. Howard JF, Bril V, Burns TM, Mantegazza R, Bilinska M, Szczudlik A, Beydoun S. Garrido FJRDR, Piehl F, Rottoli M: Randomized phase 2 study of FcRn antagonist efgartigimod in generalized myasthenia gravis. Neurology. 2019;92(23):e2661-73. 
109. Benatar M, Mcdermott MP, Sanders DB, Wolfe GI, Barohn RJ, Nowak RJ, Hehir M, Juel V, Katzberg H, Tawil R. Efficacy of prednisone for the treatment of ocular Myasthenia (EPITOME): a randomized, controlled trial. Muscle Nerve. 2016;53(3):363-9.

110. Howard FM Jr, Duane DD, Lambert EH, Daube JR. Alternate-day prednisone: preliminary report of a double-blind controlled study. Ann NY Acad Sci. 1976;274:596-607.

111. Lindberg C, Andersen O, Lefvert A. Treatment of myasthenia gravis with methylprednisolone pulse: a double-blind study. Acta Neurol Scand. 1998;97(6):370-3.

112. Sanders D, Hart I, Mantegazza R, Shukla S, Siddiqi Z, De Baets M, Melms A, Nicolle M, Solomons N, Richman DP. An international, phase III, randomized trial of mycophenolate mofetil in myasthenia gravis. Neurology. 2008;71(6):400-6.

113. Wolfe Gl, Barohn RJ, Sanders DB, McDermott MP. Comparison of outcome measures from a trial of mycophenolate mofetil in myasthenia gravis. Muscle Nerve. 2008;38(5):1429-33.

114. Group MS. A trial of mycophenolate mofetil with prednisone as initial immunotherapy in myasthenia gravis. Neurology. 2008;71(6):394-9.

115. Muppidi S. The myasthenia gravis-specific activities of daily living profile. Ann N Y Acad Sci. 2012;1274(1):114-9.

116. Muppidi S, Wolfe Gl, Conaway M, Burns TM, Composite M, Group MQS. MG-ADL: still a relevant outcome measure. Muscle Nerve. 2011:44(5):727-31.

117. Dumitru D, Amato A, Zwarts M, editors. Electrodiagnostic medicine, 2nd ed. Philadelphia: Hanley \& belfus, INC, 2002; pp. 1148-1177.

118. Jabre JF, Chirico-Post J, Weiner M. Stimulation SFEMG in myasthenia gravis. Muscle Nerve. 1989;12(1):38-42.

119. Kasper D, Fauci A, Hauser S, Longo D, Jameson J, Loscalzo J. Harrison's principles of internal medicine, 19th ed. New York: McGraw-Hill Education; 2015; pp. 2704-6.

120. Tindall RS, Rollins JT, Phillips JT, et al. Preliminary results of a doubleblind, randomized, placebo-controlled trial of cyclosporine in myasthenia gravis. N Engl J Med. 1987;316:719-24.

121. Barohn RJ, McIntire D, Herbelin L, et al. Reliability testing of the quantitative myasthenia gravis score. Ann N Y Acad Sci. 1998;841:769-72.

122. Sharshar T, Chevret S, Mazighi M, et al. Validity and reliability of two muscle strength scores commonly used as endpoints in assessing treatment of myasthenia gravis. J Neurol. 2000;247:286-90.

123. Bedlack RS, Simmel D, Bosworth $H$, et al. Quantitative myasthenia gravis score: assessment of responsiveness and longitudinal validity. Neurology. 2005;64:1968-70

124. Gilhus NE. Myasthenia and neuromuscular junction. Curr Opin Neurol. 2012;25:523-9.

125. Querol L, Illa I. Myasthenia and the neuromuscular junction. Curr Opin Neurol. 2013;26:459-65.

126. Verschuuren JJ, Huijbers MG, Plomp JJ, et al. Pathophysiology of myasthenia gravis with antibodies to the acetylcholine receptor, musclespecific kinase and low-density lipoprotein receptor-related protein 4. Autoimmun Rev. 2013;12:918-23.

127. Huijbers M, Lipka A, Plomp J, Niks E, van der Maarel S, Verschuuren J. Pathogenic immune mechanisms at the neuromuscular synapse: specific antibody-binding epitopes' role in myasthenia gravis. J Intern Med. 2014;275(1):12-26.

128. Zisimopoulou P, Brenner T, Trakas N, Tzartos SJ. Serological diagnostics in myasthenia gravis based on novel assays and recently identified antigens. Autoimmun Rev. 2013;12:924-30.

129. Verschuuren J, Strijbos E, Vincent A. Neuromuscular junction disorders. Handb Clin Neurol. 2016;133:447-66.

130. Skeie GO, Apostolski S, Evoli A, et al. Guidelines for treatment of autoimmune neuromuscular transmission disorders. Eur J Neurol. 2010;17:893-902.

131. Hart IK, Sathasivam S, Sharshar T. Immunosuppressive agents for myasthenia gravis. Cochrane Database Syst Rev. 2007;4:CD005224.

132. Brunner NG, Namba T, Grob D. Corticosteroids in management of severe, generalized myasthenia gravis. Effectiveness and comparison with corticotropin therapy. Neurology. 1972;22:603-10.

133. Mann JD, Johns TR, Campa JF, Muller WH. Long-term prednisone followed by thymectomy in myasthenia gravis. Ann NY Acad Sci. 1976;274:608-22.
134. Pascuzzi RM, Coslett HB, Johns TR. Long-term cortico- steroid treatment of myasthenia gravis: report of 116 patients. Ann Neurol. 1984;15:291-8.

135. Evoli A, Batocchi AP, Palmisani MT, Monaco ML, Tonali P. Long-term results of corticosteroid therapy in patients with myasthenia gravis. Eur Neurol. 1992;32:37-43.

136. Matell G, Baerendtz S, Hulting J, Malmlund HO. Effects on Myasthenia of twin shock doses of methylprednisolone (TSDMP). 1982. 5th Int Congr Neuromusc Diseases.

137. Arsura E, Brunner NG, Namba T, Grob D. High-dose intravenous methylprednisolone in myasthenia gravis. Arch Neurol. 1985;42:1149-53.

138. Chaudhry V, Cornblath D, Griffin J, O'Brien R, Drachman DB. Mycophenolate mofetil: a safe and promising immunosuppressant in neuromuscular diseases. Neurology. 2001;56(1):94-6.

139. Ciafaloni E, Massey J, Tucker-Lipscomb B, Sanders D. Mycophenolate mofetil for myasthenia gravis: an open-label pilot study. Neurology. 2001;56(1):97-9.

140. Keesey JC. Clinical evaluation and management of myasthenia gravis. Muscle Nerve. 2004;29:484-505.

141. Gajdos P, Chevret S, Toyka K. Plasma exchange for myas-thenia gravis. Cochrane Database Syst Rev. 2002. https://doi.org/10.1002/14651858. cd002275.

142. Insen P, Bril V. A comparison of the effectiveness of intravenous immunoglobulin and plasma exchange as preoperative therapy of myasthenia gravis. J Clin NeuromusculDis. 2008;9:352-5.

143. Ronager J, Ravnborg M, Hermansen I, Vorstrup S. Immunoglobulintreatment versus plasma exchange in patients with chronic moderate to severe myasthenia gravis. Artif Organs. 2001;25:967-73.

144. Chegini A. Therapeutic plasmapheresis in myasthenic crisis after botox injection (case report). 2016 (In Persian).

145. McLeod BC, Weinstein R, Winters JL. Textbook of apheresis principles and practice, 3rd ed. USA: AABB; 2010. pp. 295-317.

146. Cellot G, Franceschi Biagioni A, Ballerini L. Nanomedicine and graphene-based materials: advanced technologies for potential treatments of diseases in the developing nervous system. Pediatr Res. 2021. https://doi.org/10.1038/s41390-021-01681-6.

147. Ambesh P, Gregory AD. Nanotechnology in neurology: genesis, current status, and prospects. Ann Indian Acad Neurol. 2015;18(4):382-6.

148. Sriramoju B, Kanwar RK, Kanwar JR. Nanomedicine based nanoparticles for neurological disorders. Curr Med Chem. 2014;21(36):4154-68.

149. Kumar Nath U, Bhattacharyya D, Chattopadhya D, Dhingra G, Azad SH, Mohanty A. Visceral leishmaniasis masquerading as drug-induced pancytopenia in myasthenia gravis. Drug Discov Therapeutics. 2021;15(1):48-50.

150. Eftekhari A, Maleki Dizaj S, Chodari L, Sunar S, Hasanzadeh A, Ahmadian E, Hasanzadeh M. The promising future of nano-antioxidant therapy against environmental pollutants induced-toxicities. Biomed Pharmacother. 2018;103:1018-27.

\section{Publisher's Note}

Springer Nature remains neutral with regard to jurisdictional claims in published maps and institutional affiliations.

Ready to submit your research? Choose BMC and benefit from:

- fast, convenient online submission

- thorough peer review by experienced researchers in your field

- rapid publication on acceptance

- support for research data, including large and complex data types

- gold Open Access which fosters wider collaboration and increased citations

- maximum visibility for your research: over $100 \mathrm{M}$ website views per year

At BMC, research is always in progress.

Learn more biomedcentral.com/submissions 